\title{
Adaptive meshing for two-dimensional thermoelastic problems using Hermite boundary elements
}

\author{
J.C. Miranda-Valenzuela ${ }^{a}$, K.H. Muci-Küchler ${ }^{\text {b,* }}$, S. Soriano-Soriano ${ }^{c}$ \\ ${ }^{a}$ Wessex Institute of Technology, Ashurst Lodge, Ashurst, Southampton SO40 7AA, UK \\ ${ }^{\mathrm{b}}$ University of Detroit Mercy, Detroit, MI 48219-0900, USA \\ ${ }^{\mathrm{c}}$ Prolec GE, Power Division, Apodaca, N.L. 66600, Mexico
}

Received 11 April 2000; revised 3 August 2000; accepted 4 August 2000

\begin{abstract}
In the present work, error indicators for the potential and elastostatic problems are used in a combined fashion to implement an adaptive meshing scheme for the solution of two-dimensional steady-state thermoelastic problems using the Boundary Element Method. These error indicators exploit in their formulation the possibility of generating two different numerical solutions from just one analysis using Hermite elements. The first solution is the standard one obtained from an analysis using Hermite elements. The second is a "reduced" solution obtained representing the field variables inside an element using some of the degrees of freedom of the Hermite element together with Lagrangian shape functions. The basic idea behind the computation of the error indicator is to compare these two solutions, on an element by element basis, to obtain an estimate of the magnitude of the error in the numerical solution corresponding to the Hermite elements. In this sense, it is assumed that the bigger the difference between these two solutions, the bigger the error in the original solution with Hermite elements. Since the thermoelastic problem in its uncoupled fashion is considered, the former approach is applied to both problems, heat conduction and thermoelastic. Since both numerical solutions for each one of these problems are obtained from just one analysis, the computational cost of the proposed error indicators is very low. (C) 2001 Elsevier Science Ltd. All rights reserved.
\end{abstract}

Keywords: Adaptive meshing; Error estimation; Hermite boundary elements; Hypersingular boundary integral equations; Thermoelastic problems

\section{Introduction}

Despite the excellent accuracy that Hermite elements [1] can provide for the computation of the boundary stresses, it seems that this type of elements have not been able to attract enough attention from researchers and analysts in the Boundary Element Method (BEM) community. Up to now, the number of published papers dealing with the formulation, implementation, and application of Hermite elements has been relatively small. Among the references that are currently available, [2-8], provide very good examples of the advantages that Hermite elements can offer.

Two main reasons may be held accountable for the lack of popularity of Hermite elements. First, when Hermite elements are used in an isoparametric fashion, the user has to specify the unit vector tangent to the boundary at each one of the nodes in the mesh. Second, since the formulation of this type of elements requires the use of

\footnotetext{
* Corresponding author. Tel.: +1-313-993-1579; fax: +1-313-993-1187.

E-mail address: macith@udmercy.edu (K.H. Muci-Küchler).
}

the so-called Hypersingular Boundary Integral Equations (HBIEs), initially some difficulties were encountered in their numerical implementation. The first problem can be solved if, as in this work, the Hermite elements are implemented in a non-isoparametric fashion. The second one can be addressed using a regularization process for the HBIEs like the one presented by Muci-Küchler and Rudolphi [5].

The reliability of the BEM for the solution of thermoelastic problems is nowadays well recognized, and efficient implementations for steady state and transient thermoelasticity have been readily available for several years now $[9,10]$. In 1995, Muci-Küchler and Hernández-Luna [11] presented completely regularized forms of the conventional and tangent derivative boundary integral equations for the two-dimensional, steady-state, thermoelastic problem. This contribution provided a convenient alternative for the numerical implementation of Hermite elements in this type of problems.

Due to the importance of thermoelastic problems in several industries, research aimed to extend the applicability of the BEM for the solution of this type of problems still continues. One example is the development of BEM 
formulations for the analysis of thermoelastic fracture mechanics problems (see for example Refs. [12,13]).

In recent years, adaptive meshing techniques have become a topic of interest for many researchers and the number of references regarding adaptive meshing techniques in the BEM has increased significantly $[14,15]$. Nevertheless, as surprising as it may be, it seems that the thermoelastic problem has not received enough attention from the researchers in the field. In one approach that the authors are aware of, Kamiya et al. [16], used the sample point error analysis to implement adaptive meshing for this type of problems.

Another topic that apparently has not been completely addressed in the adaptive meshing field is the use of subregions. Subregions play a very important role in BEM analysis since they allow the analysis of problems in which the domain is made of dissimilar materials. Also, they provide a convenient way to model very large problems in an efficient fashion since the computational requirements for large problems decrease significantly when subregions are introduced and a sub-structuring approach is employed for their solution [10]. In adaptive meshing, the introduction of subregions posses some difficulties from the implementation point of view. This was shown by Kamiya and Koide for the case of the potential problem [17] and Kamiya et al. [18] and Muci-Küchler and Miranda-Valenzuela [19] for the elastostatic one. Nevertheless, further research in this topic would be welcomed since different approaches are possible depending on the type of problem being analyzed and the scheme used to predict the error in the solution.

Most of the research in adaptive meshing techniques is devoted to the formulation and implementation of new and more reliable error indicators. Several error indicators capable of leading an adaptive meshing process have already been published in the literature. In what follows, a brief explanation of the ones that are most closely related to this work is presented.

Charafi et al. [20] used the local reanalysis technique to implement an efficient $h$-hierarchical adaptive meshing technique. The authors employed a local enrichment scheme in order to obtain an improved solution for each one of the elements present in the mesh. The improved solution was compared with the original one to determine a measure of the error in the element.

Guiggiani [21] used the direct differentiation approach to perform a sensitivity analysis of approximate boundary element solutions with respect to the position of the collocation points. In a similar approach, Paulino et al. [22] used the rate of change of the tractions and generalized displacements with respect to the positions of the middle nodes of quadratic boundary elements as error indicators. These rates of change were calculated by the direct analytic differentiation of the governing equations for the problem under consideration. Gallego et al. [23] extended the work of Guiggiani [21] by carrying out a sensitivity analysis of the solutions for the HBIE in the potential problem. In order to obtain the sensitivity equations, the derivative with respect to the position of the collocation point of the HBIE was evaluated.

In Ref. [24], Paulino et al. proposed the evaluation of the residual of HBIEs as an error indicator. The method presented by them was developed for the approximation of the error on the boundary as well as in the interior of the domain. Liang et al. [25], used a modified version of the error indicator proposed by Paulino et al. by combining the residuals of the Conventional Boundary Integral Equations (CBIEs) and HBIEs for the computation of error indicators. Menon et al. [26] presented a basic iteration scheme that consists of using the BIEs for solving the boundary value problem and iterating this solution with the HBIEs. A residual was defined as the difference in the derivative quantities, and this residual was used as an estimate of the error in the BEM solution.

Muci-Küchler et al. [27] and Muci-Küchler and MirandaValenzuela [19] presented formulations for the computation of simple error indicators for Hermite elements in twodimensional potential and elastostatic problems. These error indicators were based on the idea of obtaining two different approximate numerical solutions for the tangential derivatives of the field variables from just one analysis with Hermite elements. The comparison of the two approximate solutions inside an element was used as a measure of the error for that element.

In this work, the ideas presented in Refs. [19,27] are extended to perform adaptive meshing with Hermite elements in steady-state, uncoupled, two-dimensional thermoelastic problems. Since the thermoelastic problem in its uncoupled fashion is considered, it is possible to solve the heat conduction and thermoelastic problems separately, solving first for the temperature distribution on the boundary, and then using that information to solve for the boundary stresses. This allows to estimate the error in the solution for the heat conduction and thermoelastic problems in a separate fashion, giving some flexibility to the adaptive process.

Also, this paper discusses some of the schemes that are currently available to carry out the mesh refinement process in steady-state, uncoupled, two-dimensional thermoelastic problems and presents new alternatives to improve them. Special attention is given on how to implement these schemes in models with subregions, since additional considerations are required in those cases, especially when subregions that have different physical properties are involved. Furthermore, a more general approach than the one given in Ref. [27] for the computation of the error indicator for the potential problem is also presented, tackling some of the restrictions given in that reference. Numerical examples are included to illustrate the versatility of the formulation and its numerical implementation.

\section{BEM formulation}

As will be discussed later, in order to formulate Hermite 


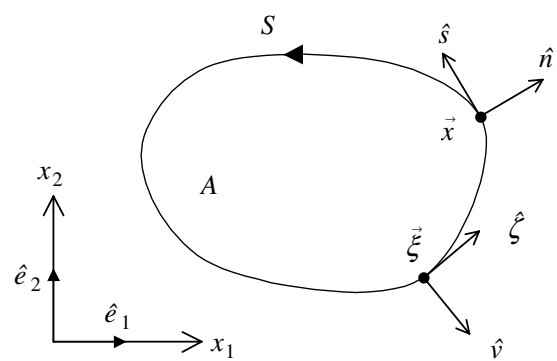

Fig. 1. Problem domain, unit vectors, and global coordinate system.

elements, the CBIEs and the Tangent Derivative Boundary Integral Equations (TDBIEs) must be simultaneously collocated at the same points on the boundary. This coincident collocation, together with the use of Hermite shape functions, provides very accurate results for the stresses on the boundary [5] and, at the same time, allows the possibility to obtain an inexpensive error indicator.

In this section, the CBIEs and the TDBIEs for the heat conduction and thermoelastic problems are presented. In addition, a brief review on how Hermite elements are formulated and how the boundary stresses can be computed is presented.

\subsection{Boundary integral equations for the heat conduction problem}

Consider the two-dimensional, steady-state, heat conduction problem without interior heat generation. For a domain $A$ enclosed by a boundary $S$, the CBIE and the TDBIE, both in a completely regularized form, can be written as [4]:

$$
\begin{aligned}
& \int_{S} G(\vec{x}, \vec{\xi})[T(\vec{x})-T(\vec{\xi})] \mathrm{d} S(\vec{x})=\int_{S} F(\vec{x}, \vec{\xi}) q(\vec{x}) \mathrm{d} S(\vec{x}) \\
& \int_{S} V(\vec{x}, \vec{\xi})\left[T(\vec{x})-T(\vec{\xi})-\frac{\partial T}{\partial \zeta}(\vec{\xi}) r_{j} \zeta_{j}\right] \mathrm{d} S(\vec{x}) \\
& -\int_{S} Y(\vec{x}, \vec{\xi}) \mathrm{d} S(\vec{x}) \frac{\partial T}{\partial \zeta}(\xi) \\
& =\int_{S}\left[W^{0}(\vec{x}, \vec{\xi}) q(\vec{x})-W(\vec{x}, \vec{\xi}) q(\vec{\xi})\right] \mathrm{d} S(\vec{x})
\end{aligned}
$$

where $T$ represents the temperature, $q$ represents the normal heat flux, $\vec{x}$ and $\vec{\xi}$ are the field and source points, respectively, and $\zeta_{i}$ are the components of the unit vector tangent to the boundary at $\vec{\xi}$ (see Fig. 1). Also, in the above equations and in the ones to follow, unless specified otherwise, the range of the indices goes from 1 to 2 , and the summation convention is employed.

The kernels $F, G, V, Y, W$, and $W^{o}$, that appear in the two boundary integral equations under consideration are given by:

$$
\begin{aligned}
& F(\vec{x}, \vec{\xi})=-\frac{1}{2 \pi k} \ln (r) \\
& G(\vec{x}, \vec{\xi})=-k \frac{\partial F(\vec{x}, \vec{\xi})}{\partial n}=\frac{1}{2 \pi r} \hat{e}_{r} \cdot \hat{n}
\end{aligned}
$$

$$
\begin{aligned}
& V(\vec{x}, \vec{\xi})=\zeta_{i} \frac{\partial G(\vec{x}, \vec{\xi})}{\partial \xi_{i}} \\
& Y(\vec{x}, \vec{\xi})=k \frac{\partial F(\vec{x}, \vec{\xi})}{\partial \xi_{i}} \zeta_{i} \zeta_{j} n_{j} \\
& W(\vec{x}, \vec{\xi})=s_{i} \frac{\partial F(\vec{x}, \vec{\xi})}{\partial \xi_{i}}
\end{aligned}
$$

$$
W^{0}(\vec{x}, \vec{\xi})=\zeta_{i} \frac{\partial F(\vec{x}, \vec{\xi})}{\partial \xi_{i}}
$$

In the above expressions, $r$ is the distance between the source and field points, $s_{i}$ and $n_{i}$ are the components of the unit vectors tangent and normal to the boundary at $\vec{x}, \vec{r}=\vec{x}-\vec{\xi}$, and $\hat{e}_{r}=$ $\vec{r} / r$.

\subsection{Boundary integral equations for the thermoelastic problem}

Consider the two-dimensional, plane strain, steady-state, thermoelastic problem without body forces. Furthermore, assume that the material obeys the Duhamel-Neumann generalization of Hooke's law and that no heat generation takes place inside the domain. For a two-dimensional domain $A$ bounded by a curve $S$, the CBIEs corresponding to this problem, in a completely regularized form, can be written as [11]:

$$
\begin{aligned}
& \int_{S} T_{i j}(\vec{x}, \vec{\xi})\left[u_{j}(\vec{x})-u_{j}(\vec{\xi})\right] \mathrm{d} S(\vec{x}) \\
& =\int_{S} U_{i j}(\vec{x}, \vec{\xi}) t_{j}(\vec{x}) \mathrm{d} S(\vec{x})+\int_{S} M_{i}(\vec{x}, \vec{\xi}) T(\vec{x}) \mathrm{d} S(\vec{x}) \\
& \quad+\int_{S} N_{i}(\vec{x}, \vec{\xi}) \frac{\partial T(\vec{x})}{\partial n} \mathrm{~d} S(\vec{x})
\end{aligned}
$$

where $u_{i}$ and $t_{i}$ are the displacement and traction components, and $n$ represents the direction of the unit outward normal vector at the field point. The kernels $U_{i j}$ and $T_{i j}$ are the standard displacement and traction kernels given by fundamental solutions for the elastostatic problem in twodimensions, and $N_{i}$ and $M_{i}$ are the kernels product of the introduction of the thermal loading. For plane strain conditions, these kernels are given by (see for example Ref. [9]):

$$
\begin{aligned}
U_{i j}(\vec{x}, \vec{\xi})= & \frac{-1}{8 \pi \mu(1-\nu)}\left[(3-4 \nu) \delta_{i j} \ln r-r_{, i} r_{, j}\right] \\
T_{i j}(\vec{x}, \vec{\xi})= & \frac{-1}{4 \pi(1-\nu)}\left(\frac{1}{r}\right)\left\{\left[(1-2 \nu) \delta_{i j}+2 r_{, i} r_{, j}\right] \frac{\partial r}{\partial n}\right. \\
& \left.-(1-2 \nu)\left(r_{, i} n_{j}-r_{, j} n_{i}\right)\right\}
\end{aligned}
$$


$N_{i}(\vec{x}, \vec{\xi})=\frac{\alpha(1+\nu)}{8 \pi(1-\nu)}[1+2 \ln r] r_{i}$

$M_{i}(\vec{x}, \vec{\xi})=\frac{-\alpha(1+\nu)}{8 \pi(1-\nu)}\left[n_{i}(1+2 \ln r)+2 r_{, i} r_{, j} n_{j}\right]$

where $\mu$ is the shear modulus, $\nu$ is Poisson's ratio, $\alpha$ is the thermal expansion coefficient, and $r_{i}$ are the components of the position vector $\vec{r}$.

As was mentioned previously, in addition to the CBIEs, the TDBIEs must also be collocated at the same points on the boundary to formulate Hermite elements. These equations are obtained taking the derivative of the displacement representation integral with respect to the source point coordinates $\xi_{k}$, contracting the resulting expression with $\zeta_{k}$, and taking the source point to the boundary. The resulting equations, in their regularized form, can be written as [11]:

$$
\begin{aligned}
& \int_{S} V_{i j}(\vec{x}, \vec{\xi})\left[u_{j}(\vec{x})-u_{j}(\vec{\xi})-\frac{\partial u_{j}}{\partial \zeta}(\vec{\xi}) r_{m} \zeta_{m}\right] \mathrm{d} S(\vec{x}) \\
& +\int_{S} Y_{i j}(\vec{x}, \vec{\xi}) \mathrm{d} S(\vec{x}) \frac{\partial u_{j}}{\partial \zeta}(\vec{\xi}) \\
& =\int_{S}\left[W_{i j}^{0}(\vec{x}, \vec{\xi}) t_{j}(\vec{x})-W_{i j}(\vec{x}, \vec{\xi}) t_{j}(\vec{\xi})\right] \mathrm{d} S(\vec{x})+\int_{S} Z_{i}(\vec{x}, \vec{\xi}) \\
& \quad \times[T(\vec{x})-T(\vec{\xi})] \mathrm{d} S(\vec{x})+\int_{S} Z_{i}^{0}(\vec{x}, \vec{\xi}) \mathrm{d} S(\vec{x}) T(\vec{\xi}) \\
& \quad+\int_{S} Z_{i}^{1}(\vec{x}, \vec{\xi}) \frac{\partial T(\vec{x})}{\partial n} \mathrm{~d} S(\vec{x})
\end{aligned}
$$

In the above expression, the kernels $V_{i j}, Y_{i j}, W_{i j}, W_{i j}^{0}, Z_{i}, Z_{i}^{0}$ and $Z_{i}^{1}$, are given by:

$$
\begin{aligned}
V_{i j}(\vec{x}, \vec{\xi})= & \zeta_{k} \frac{\partial T_{i j}(\vec{x}, \vec{\xi})}{\partial \xi_{k}} \\
Y_{i j}(\vec{x}, \vec{\xi})= & \mu\left\{\frac{2 \nu}{1-2 \nu} \zeta_{j}\left(\zeta_{k} n_{l}-s_{k} v_{l}\right) \frac{\partial U_{i l}(\vec{x}, \vec{\xi})}{\partial \xi_{k}}\right. \\
& \left.+\zeta_{k} n_{l} \zeta_{l} \frac{\partial U_{i j}(\vec{x}, \vec{\xi})}{\partial \xi_{k}}+\zeta_{l}\left(\zeta_{k} n_{j}-s_{k} v_{j}\right) \frac{\partial U_{i j}(\vec{x}, \vec{\xi})}{\partial \xi_{k}}\right\}
\end{aligned}
$$

$W_{i j}(\vec{x}, \vec{\xi})=s_{k} \frac{\partial U_{i j}(\vec{x}, \vec{\xi})}{\partial \xi_{k}}$

$$
W_{i j}^{0}(\vec{x}, \vec{\xi})=\zeta_{k} \frac{\partial U_{i j}(\vec{x}, \vec{\xi})}{\partial \xi_{k}}
$$

$Z_{i}(\vec{x}, \vec{\xi})=\zeta_{k} \frac{\partial M_{i}(\vec{x}, \vec{\xi})}{\partial \xi_{k}}$

$$
Z_{i}^{0}(\vec{x}, \vec{\xi})=\frac{\alpha(1+\nu)}{2 \pi(1-\nu)} \frac{r_{, k}}{r}\left[\zeta_{k} n_{i}-s_{k} \nu_{i}\right]
$$

$Z_{i}^{1}(\vec{x}, \vec{\xi})=\zeta_{k} \frac{\partial N_{i}(\vec{x}, \vec{\xi})}{\partial \xi_{k}}$

where $v_{k}$ and $\zeta_{k}$ are the components of the unit normal and tangent vectors at $\vec{\xi}$ (see Fig. 1).

For the numerical implementation of the CBIEs and TDBIEs corresponding to the heat conduction and the thermoelastic problems, the integrals over the boundary are divided into integrals over boundary segments or "boundary elements". In addition, the temperature, the normal heat flux, the displacements, and the tractions inside each element are approximated using Hermite's interpolation polynomials.

\subsection{Hermite elements}

Since the introduction of Hermite boundary elements in 1986 by Watson [1], this kind of elements has been successfully employed in many applications. Hermite elements use Hermite interpolation polynomials as shape functions, and employ the nodal values of the field variables and their tangential derivatives as the degrees of freedom associated with the functional nodes of the element.

In this work, all the field variables involved, namely $T, q$, $u_{i}$, and $t_{i}$, are represented inside each element in the same fashion. If we let $w$ represent either $T, q, u_{i}$, or $t_{i}$, inside each element, $w$ is approximated as follows:

$w(\eta)=\sum_{k=1}^{\mathrm{NN}}\left[H_{1}^{(k)} w^{(k)}(\eta)+H_{2}^{(k)} \frac{\partial w^{(k)}}{\partial s}\right]$

where $\mathrm{NN}$ is the total number of nodes in the element (usually 2$), H_{i}^{(k)}(i=1,2)$ are the shape functions associated with the local node $k$, and $\eta \in[-1,1]$ is the local coordinate on a master element.

The shape functions $H_{i}^{(k)}$ for a continuous cubic Hermite element are given by:

$H_{1}^{(1)}(\eta)=(1-\eta)^{2}(2+\eta) / 4$

$H_{2}^{(1)}(\eta)=\partial S /\left.\partial \eta\right|_{(1)}(1-\eta)^{2}(1+\eta) / 4$

$H_{1}^{(2)}(\eta)=(1+\eta)^{2}(2-\eta) / 4$

$H_{2}^{(2)}(\eta)=-\partial S /\left.\partial \eta\right|_{(2)}(1+\eta)^{2}(1-\eta) / 4$

where the second and fourth shape functions contain the reduced Jacobian of the geometric transformation evaluated at the local functional nodes located at $\eta=-1$ and $\eta=1$, respectively.

On a curvilinear element in two dimensions, the reduced Jacobian is given by

$J=\frac{\partial S}{\partial \eta}=\sqrt{\left(\frac{\mathrm{d} x_{1}}{\mathrm{~d} \eta}\right)^{2}+\left(\frac{\mathrm{d} x_{2}}{\mathrm{~d} \eta}\right)^{2}}$

where the derivatives $\mathrm{d} x_{1} / \mathrm{d} \eta$ and $\mathrm{d} x_{2} / \mathrm{d} \eta$ are determined through the geometric representation used for the element. 
Therefore, if as in this work, Lagrangian elements are used to approximate the geometry,

$$
\frac{\mathrm{d} x_{i}}{\mathrm{~d} \eta}=\sum_{k=1}^{\mathrm{NNG}} \frac{\partial}{\partial \eta} \hat{H}_{k}(\eta) x_{i}^{(k)}
$$

where NNG is the total number of geometric nodes in the element, and $\hat{H}_{k}(k=i, \ldots, \mathrm{NNG})$ are the Lagrangian shape functions associated with the element.Since in a well-posed two-dimensional problem Hermite elements have twice the number of unknown nodal values than Lagrangian elements, it is necessary to collocate two different sets of equations at each functional node in order to be able to solve for all the unknowns. These equations are the CBIEs and the TDBIEs presented previously. This coincident collocation is possible since the CBIEs and the TDBIEs are numerically independent.

One inconvenience associated with the use of the TDBIEs is that they cannot be collocated at points on the boundary where the tangential derivatives of the field variables are not continuous, corners being the most common example of such points. As many practical problems involve this type of situation, elements capable of handling it are needed. One simple solution is to move the nodes of the Hermite element towards the interior of the element where the required continuity is guaranteed. For the sake of completeness, the shape functions for partially discontinuous and discontinuous cubic Hermite elements with two nodes are presented below.

The shape functions for the partially discontinuous element with local functional nodes located at $\eta=-1$ and $\eta=1 / 2$ are given by:

$H_{1}^{(1)}=(1-2 \eta)^{2}(7+4 \eta) / 27$

$H_{2}^{(1)}=\partial S /\left.\partial \eta\right|_{(1)}(1-2 \eta)^{2}(1+\eta) / 9$

$H_{1}^{(2)}=4(1+\eta)^{2}(5-4 \eta) / 27$

$H_{2}^{(2)}=-2 \partial S /\left.\partial \eta\right|_{(2)}(1+\eta)^{2}(1-2 \eta) / 9$

For the discontinuous element with local functional nodes at $\eta=-1 / 2$ and $\eta=1 / 2$, the shape functions are given by:

$H_{1}^{(1)}=(1-2 \eta)^{2}(1+\eta) / 2$

$H_{2}^{(1)}=\partial S /\left.\partial \eta\right|_{(1)}(1-2 \eta)^{2}(1+2 \eta) / 8$

$H_{1}^{(2)}=(1+2 \eta)^{2}(1-\eta) / 2$

$H_{2}^{(2)}=-\partial S /\left.\partial \eta\right|_{(2)}(1+2 \eta)^{2}(1-2 \eta) / 8$

\subsection{Computation of stresses}

Consider a model where an approximation for the displacements and the tractions inside each element is available from the BEM solution. The first step in the computation of the stresses is the evaluation of the in- plane components of the stress tensor in terms of a local coordinate system. At a boundary point $\vec{x}$ the normal stress in the normal direction, the shear stress, and normal stress in the tangential direction, can be obtained for plane strain conditions as follows:

$\sigma_{11}^{\prime}=t_{1}^{\prime} \quad \sigma_{12}^{\prime}=t_{2}^{\prime}$

$\sigma_{22}^{\prime}=\frac{1}{1-\nu}\left(\nu \sigma_{11}^{\prime}+2 \mu \epsilon_{22}^{\prime}-2 \mu(1+\nu) \alpha T\right)$

As can be observed, the computation of $\sigma_{11}^{\prime}$ and $\sigma_{12}^{\prime}$ is straightforward since the values for the tractions are available from the BEM solution or the boundary conditions, and the normal and tangent unit vectors can be easily obtained from the geometric representation of the boundary. However, $\sigma_{22}^{\prime}$ requires the prior computation of the normal strain in the tangential direction $\epsilon_{22}^{\prime}$ which is given by:

$\epsilon_{22}^{\prime}=\frac{\partial u_{2}^{\prime}}{\partial x_{2}^{\prime}}$

where $\partial u_{2}^{\prime} / \partial x_{2}^{\prime}=\left(\partial u_{i} / \partial s\right) s_{i}$ are the tangential derivatives of the displacement components. If the values of $\partial u_{i} / \partial s$ at the functional nodes are not directly available from the solution of the system of equations generated by the BEM, they need to be computed through the differentiation of the functional representation for the displacements corresponding to the element. After the values of the in-plane stress components in the local coordinate system have been obtained, they can be easily transformed into the global coordinate system using the well-known transformation formula for secondorder tensors:

$\left[\begin{array}{l}\sigma_{11} \\ \sigma_{22} \\ \sigma_{12}\end{array}\right]=\left[\begin{array}{ccc}n_{1}^{2} & n_{2}^{2} & -2 n_{1} n_{2} \\ n_{2}^{2} & n_{1}^{2} & 2 n_{1} n_{2} \\ n_{1} n_{2} & -n_{1} n_{2} & n_{1}^{2}-n_{2}^{2}\end{array}\right]\left[\begin{array}{l}\sigma_{11}^{\prime} \\ \sigma_{22}^{\prime} \\ \sigma_{12}^{\prime}\end{array}\right]$

where $n_{i}$ are the components of the unit outward normal vector at the point of interest.

\section{Formulation of the error indicators}

One of the ideas that is commonly employed to formulate error indicators in different numerical methods, is to use the difference between two numerical solutions with different number of degrees of freedom to obtain an approximate measure of the error in the most accurate of the two.

Assume that a sequence of approximate solutions for the displacements $\hat{u}_{i}^{\{1\}}(\vec{x}), \hat{u}_{i}^{\{2\}}(\vec{x}), \hat{u}_{i}^{\{3\}}(\vec{x})$ etc. for a given problem is available, and that each one of them has more degrees of freedom than the previous one. Let $\hat{\sigma}_{i j}^{\{1\}}(\vec{x})$, $\hat{\sigma}_{i j}^{\{2\}}(\vec{x}), \hat{\sigma}_{i j}^{\{3\}}(\vec{x})$, etc. be the stresses corresponding to the approximate solutions for the displacements. As the number of degrees of freedom increases, it is reasonable to expect that the resulting numerical solution will get closer to the exact solution if the method is converging to the latter. That 


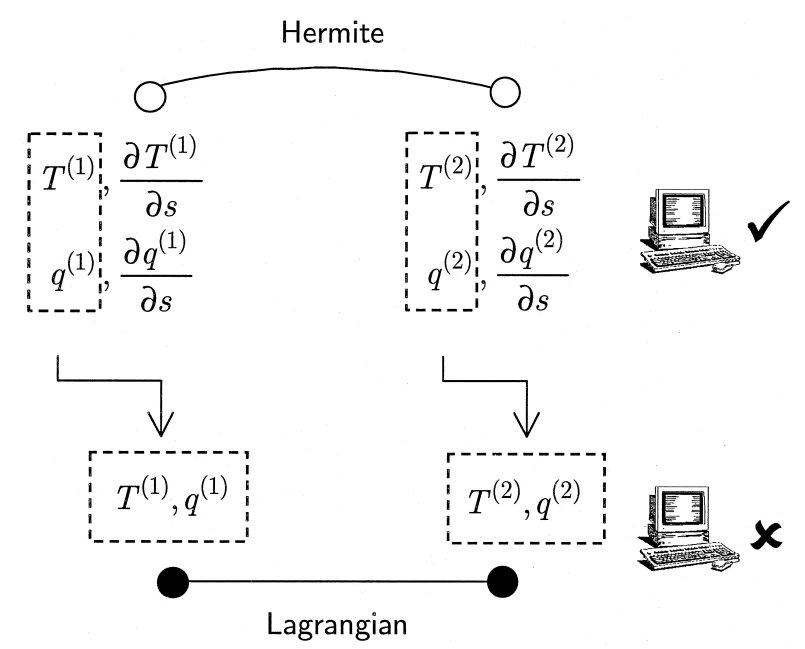

Fig. 2. Obtention of the second "reduced" solution from the solution withHermite elements in the potential problem.

is, if the sequence will converge to the exact solution as the number of degrees of freedom increases indefinitely, then:

$\left|\sigma_{i j}(\vec{x})-\hat{\sigma}_{i j}^{\{n\}}(\vec{x})\right| \rightarrow 0$ as $n \rightarrow \infty$

where $\sigma_{i j}(\vec{x})$ is the analytical solution and $\hat{\sigma}_{i j}^{\{n\}}(\vec{x})$ corresponds to a numerical solution that has more degrees of freedom than $\hat{\sigma}_{i j}^{\{n-1\}}(\vec{x})$.

If this is the case, then two different approximate solutions in the sequence must approach arbitrarily close to each other, that is

$\left|\hat{\sigma}_{i j}^{\{m\}}(\vec{x})-\hat{\sigma}_{i j}^{\{n\}}(\vec{x})\right| \rightarrow 0$ as $n, m \rightarrow \infty$

since

$$
\begin{aligned}
& \left|\sigma_{i j}(\vec{x})-\hat{\sigma}_{i j}^{\{n\}}(\vec{x})\right|+\left|\sigma_{i j}(\vec{x})-\hat{\sigma}_{i j}^{\{m\}}(\vec{x})\right| \\
& \quad \geq\left|\left(\sigma_{i j}(\vec{x})-\hat{\sigma}_{i j}^{\{n\}}(\vec{x})\right)-\left(\sigma_{i j}(\vec{x})-\hat{\sigma}_{i j}^{\{m\}}(\vec{x})\right)\right| \\
& \quad=\left|\hat{\sigma}_{i j}^{\{m\}}(\vec{x})-\hat{\sigma}_{i j}^{\{n\}}(\vec{x})\right|
\end{aligned}
$$

The two solutions with different number of degrees of freedom can be chosen almost arbitrarily as long as they fulfil the basic requirements for convergence. One convenient approach is to choose any first solution and select as the second one, one with twice the number of degrees of freedom. This approach has been successfully used by Rencis and Mullen [28,29] and more recently by Rodríguez and Power [30], both using Lagrangian elements to obtain both solutions. Another possible approach is to use Lagrangian elements to obtain the first solution and then use Hermite elements to obtain the second one. For elements having the same number of functional nodes, in two-dimensional problems the Hermite elements have twice the number of degrees of freedom than the Lagrangian ones, making the comparison possible.

Despite the effectiveness of using the difference between two numerical solutions to obtain a measure of the error, the use of this technique could be inefficient when implementing it as part of an adaptive meshing scheme. As two BEM solutions are needed at every step of the adaptive process, the computational time involved could become excessive. At some extent, some proposals exist to reduce this computational cost. One is the use of $h$-hierarchical functions (see Ref. [20]). Another approach is to consider that the desired values of the solution on all those elements whose computed error is below a certain prescribed value are obtained from previous analyses (see Ref. [30]).

For certain problems, or under some circumstances, it may be more useful to measure the error in the tangential derivatives of the field variables rather than in the field variables themselves. In those cases, Muci-Küchler et al., [27] and Muci-Küchler and Miranda-Valenzuela [19] have shown that when Hermite elements are used, it is possible to eliminate the need of performing two different BEM solutions at each step of the adaptive process. Since the Hermite elements involve in their formulation the nodal values of the field variables and their tangential derivatives, it is possible to obtain two different approximate solutions for the tangential derivatives from only one analysis with Hermite elements. The first solution is obtained through the use of the functional representation for the Hermite elements, which contains the nodal values of the tangential derivatives of the field variables that were obtained directly from the BEM. The second one is obtained through the use of the nodal values of the field variables and the differentiation of Lagrangian shape functions.

This procedure of comparing the two solutions obtained from one analysis with Hermite elements could be seen, in a sense, as 'equivalent' to solve a model composed of Lagrangian elements, 'enriching' the elements to become Hermite elements, and solving the new model. Then, the results for the tangential derivatives of the field variables, or associated quantities such as stresses, obtained from both models are compared on an 'element by element' basis. An advantage of the approach used here is that the absence of an analysis with Lagrangian elements makes the computation of the error indicator extremely fast. Fig. 2 shows this idea graphically for the case of the heat conduction problem.

The numerical solution of the problem under consideration is done in two parts since the heat conduction and thermoelastic equations are solved in two different analyses. The heat conduction problem is solved first. Then, its results are substituted into the thermoelastic model and the latter is solved. This gives the opportunity to use independent error indicators for each problem.

\subsection{Heat conduction problem}

For the heat conduction problem, the idea behind the error indicator is to measure the difference between the two numerical solutions for the value of the tangential derivative of the temperature or the normal heat flux, depending on the 
boundary conditions imposed on the element (see Ref. [27] for more details). As a rule, always the difference will be measured in terms of the quantity that was obtained from the solution of the system of equations generated by the BEM.

Let $m^{h}$ denote the value of the tangential derivative of $w$ ( $w$ being the temperature or normal heat flux) for the standard solution with Hermite elements. For a given point $\eta$ inside an element $(\eta \in[-1,1])$, the value of $m^{h}(\eta)$ can be computed as follows:

$$
\begin{aligned}
m^{h}(\eta) & =\frac{\partial w}{\partial s}(\eta) \\
& =\frac{1}{J(\eta)} \sum_{n=1}^{\mathrm{NN}}\left[\frac{\partial H_{n}^{(1)}(\eta)}{\partial \eta} w^{(n)}+\frac{\partial H_{n}^{(2)}(\eta)}{\partial \eta}\left(\frac{\partial w^{(n)}}{\partial s}\right)\right]
\end{aligned}
$$

where $\mathrm{NN}$ is the number of functional nodes in the element, $w^{(n)}$ is the value of temperature or the normal heat flux at node $n$, and $J(\eta)$ is the reduced Jacobian of geometric transformation.

In a similar way, for the solution with Lagrangian elements, the value of the tangential derivative of $w$ at point $\eta$, namely $m^{l}(\eta)$, can be expressed as follows:

$m^{l}(\eta)=\frac{\partial w}{\partial s}(\eta)=\frac{1}{J(\eta)} \sum_{n=1}^{\mathrm{NN}}\left(\frac{\partial \hat{H}_{n}(\eta)}{\partial \eta} w^{(n)}\right)$

where $\hat{H}_{n}$ is the Lagrangian shape function associated with node $n$.

It is important to recall that if the point of interest is located at a functional node, the Hermite solution does not require the use of Eq. (34). This is because the values of $\partial w / \partial s$ at the functional nodes are obtained directly from the BEM solution. However, for the solution with Lagrangian elements, Eq. (33) is always used since these elements do not provide the values of $\partial w_{n} / \partial s$ directly.

One convenient way of measuring the difference between $m^{h}(\eta)$ and $m^{l}(\eta)$ on an element is to compute, in the $L_{2}$ norm, the difference between both quantities

$\lambda_{m}^{(e)}=\left\{\int_{-1}^{1}\left[m^{h}(\eta)-m^{l}(\eta)\right]^{2} J(\eta) \mathrm{d} \eta\right\}^{1 / 2}$

Another way of measuring the difference between $m^{h}$ and $m^{l}$ on an element is to compute the $l_{2}$ norm of the values of that difference at a discrete number of sample points distributed along the element. In this way, the error indicator is given by

$\lambda_{m}^{(e)}=\sqrt{\sum_{k=1}^{K}\left[m^{h}\left(\eta_{k}\right)-m^{l}\left(\eta_{k}\right)\right]^{2}}$

where $K$ is the number of sample points selected, and $\eta_{k}$ is the local coordinate associated with the $k$ th sample point.

This formulation is a general case of the one presented in Ref. [27] since it is suitable for Hermite elements with more than two nodes. As expected, it can be shown that Eq. (37) reduces to the expressions presented in that reference for the case of Hermite elements with two nodes.

The choice of using either summations or integrals to compute the error indicators is basically given in terms of computational cost. The use of integrals is usually more robust but in general involves a higher computational cost. The use of summations usually offers a lower computational cost, but some reliability in the computation of the error indicator may be compromised. For most analyses, the use of error indicators based on integrals computed using from 6 to 8 Gaussian points seems to provide a good balance between reliability and cost. All the examples presented in this work were performed under this scheme. If the reader is interested in reviewing some results obtained through the summation approach, Muci-Küchler et al. [27], Muci-Küchler and Miranda-Valenzuela [19], and Miranda-Valenzuela et al. [31] present some examples for the potential, elastostatic, and thermoelastic problems, respectively.

\subsection{Thermoelastic problem}

From Eq. (28) it is clear that for the thermoelastic problem the computation of the stresses depends on the following quantities: the unit normal and tangent vectors at the point of interest, two tractions, two tangential derivatives of the displacements, and the temperature. Since the unit normal and tangent vectors depend only on the geometry of the boundary, they will be the same for both solutions because they share the same geometric representation. On the other hand, in general, the values of the tractions, tangential derivatives of the displacements, and temperature, will be different since the computation of those quantities requires the use of the shape functions. The only case in which the tractions and the temperature will have the same values is when the point of interest is located at a functional node since the use of the shape functions is not required for their computation.

Recalling the ideas presented for the heat conduction problem, it is equally possible to obtain two different numerical solutions for the stresses from a single analysis with Hermite elements. Again, the first solution is the standard solution with Hermite elements, whereas the second one is obtained using the conventional degrees of freedom of the Hermite element (displacements, tractions, and temperature) together with Lagrangian shape functions (see Fig. 3).

Hermite elements recover directly from the BEM solution the nodal values of $\partial u_{i} / \partial s$. If the values for the tangential derivatives of the displacements are needed at locations other than the functional nodes, they can be obtained through the differentiation of the Hermitian shape functions:

$$
\frac{\partial u_{i}(\eta)}{\partial s}=\frac{1}{J(\eta)} \sum_{n=1}^{\mathrm{NN}}\left(\frac{\partial H_{1}^{(n)}(\eta)}{\partial \eta} u_{i}^{(n)}+\frac{\partial H_{2}^{(n)}(\eta)}{\partial \eta} \frac{\partial u_{i}^{(n)}}{\partial s}\right)
$$

For the Lagrangian solution, the values for the tangential derivatives of the displacements must always be computed 


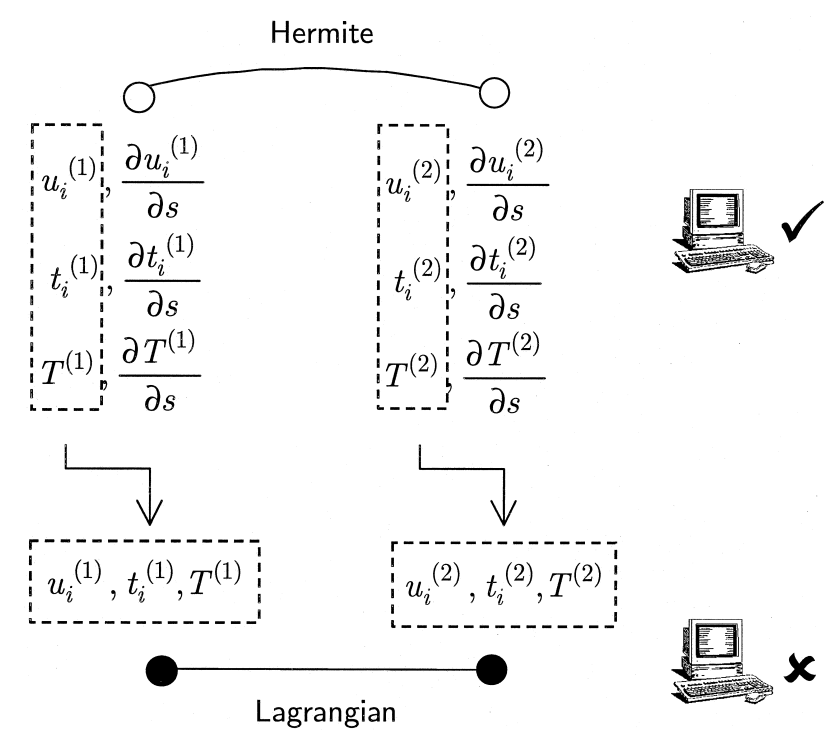

Fig. 3. Obtention of the second "reduced" solution from the solution with Hermite elements in the thermoelastic problem.

through the differentiation of the shape functions:

$$
\frac{\partial u_{i}}{\partial s}=\frac{1}{J(\eta)} \sum_{n=1}^{\mathrm{NN}}\left(\frac{\partial \hat{H}_{n}(\eta)}{\partial \eta} u_{i}^{(n)}\right)
$$

The tractions must be computed through their functional representation only if the point of interest is not a functional node. Hence,

$t_{i}(\eta)=\sum_{n=1}^{\mathrm{NN}}\left(H_{1}^{(n)}(\eta) t_{i}^{(n)}+H_{2}^{(n)}(\eta) \frac{\partial t_{i}^{(n)}}{\partial s}\right)$

for the Hermite solution, and

$t_{i}(\eta)=\sum_{n=1}^{\mathrm{NN}}\left(\hat{H}_{n}(\eta) t_{i}^{(n)}\right)$

for the Lagrangian solution.

Finally, the temperature $T$ has to be computed in a similar way as the tractions if the point of interest does not lie at a functional node.

Denoting the values of the stresses computed through the Hermite solution as $\sigma^{h}$ and the values of the stresses computed through the Lagrangian elements as $\sigma^{l}$, the error indicator for the element can be computed using the $L_{2}$ norm as:

$\lambda_{\sigma}^{(e)}=\left\{\int_{-1}^{1}\left[\sigma^{h}(\eta)-\sigma^{l}(\eta)\right]^{2} J(\eta) \mathrm{d} \eta\right\}^{1 / 2}$

Of course, the $l_{2}$ norm can be used instead if desired.

Until now, nothing has been said about which specific stress component is considered in Eq. (42). Although any stress component can be used, it must be mentioned that, in general, different stresses may lead to different results. This opens the possibility for choosing a specific stress component if the analyst is particularly interested in the error in that specific component of the stress tensor. Furthermore, the user could measure the error in the von Mises stress, the maximum shear stress, or the maximum or minimum principal stress. These stresses are commonly used in the design of mechanical components.

\section{Adaptive meshing implementation}

\subsection{Mesh improvement}

Once the error indicators $\lambda_{m}^{(e)}$ for the heat conduction problem and $\lambda_{\sigma}^{(e)}$ for the thermoelastic one have been defined, the next step is to specify how the mesh will be improved and under which criteria.

In essence, the decision of when to improve an element is based solely on the comparison of the value for its error indicator against a predetermined reference value selected by the user. This reference value can be either an absolute value or a relative one. An absolute reference value is one that is specified without using the values of the error indicator for the elements in the mesh. However, a relative reference value is one that is established based on the values of the error indicator for the elements in the mesh.

Two examples of absolute reference values are: $5 \%$ of the maximum applied traction, and $1 \%$ of the maximum value expected for the von Mises stress on the model. Examples of relative reference values are: 0.7 times the maximum value for the error indicator for the elements in the mesh, and the average value for the error indicator for the elements in the mesh.

In case the user/analyst wants to specify a relative reference value, in some cases it is useful to obtain an average index using the values for the error indicator corresponding to each one of the elements in the mesh. The average index can be obtained as:

$\tau=\frac{1}{\mathrm{NE}} \sum_{e=1}^{\mathrm{NE}} \lambda^{(e)}$

where NE is the total number of elements in the mesh.

In the same manner, it can be useful to normalize the values for the error indicator with respect to the maximum value for this quantity in the mesh:

$\hat{\lambda}^{(e)}=\frac{\lambda^{(e)}}{\max \left(\lambda^{(e)}\right)}$

From the previous discussion, three basic schemes to decide when to improve an element could be considered:

- Improve all those elements whose value for $\lambda^{(e)}$ is greater than the average index $\tau$.

- Compare the value of $\hat{\lambda}^{(e)}$ with a constant $\alpha$ in the range $[0,1]$ and improve all those elements whose value of $\hat{\lambda}^{(e)}$ is greater than $\alpha$.

- Compare the value of $\lambda^{(e)}$ with a given reference value and improve all those elements with an error indicator greater than the specified reference value. 
Since in this work the heat conduction analysis is carried out first and the thermoelastic analysis is carried out later, the improvement criterion has to be applied twice, once for each problem.

It is important to notice that if the model has subregions, it may be necessary to do additional computations to select which elements are to be improved. This is true especially for elements lying on an interface between subregions that have different properties as more than one value for the error indicator will be available.

In what follows, the different cases are discussed in detail.

\subsubsection{Heat conduction problem}

The heat conduction problem presents some difficulties as error indicators can be given in terms of the tangential derivatives of temperature or the normal heat flux, two quantities that cannot be compared directly.

For convenience, in what follows the error indicators based on $\partial T / \partial s$ will be denoted as $\lambda_{T}$ whereas the error indicators based on $\partial q / \partial s$ will be denoted as $\lambda_{q}$. As both errors have different units, in general two reference values should be given, one for each error indicator (it should be noted however, that is possible to treat both errors in an unified manner [17]).

Four cases are to be considered:

1. The reference values are absolute and the element is lying on the boundary. In this case there is only one value to be considered, $\lambda_{T}$ or $\lambda_{q}$, depending on the boundary conditions imposed on the element.

2. The reference values are absolute and the element is lying on an interface. Here, two values are available: $\lambda_{T}$ and $\lambda_{q}$. First, both errors are compared with their corresponding reference value. The one with the larger relative value will be taken as representative for the element.

3. The reference values are relative and the element is lying on the boundary. In this case, the error to be considered may be $\lambda_{T}$ or $\lambda_{q}$ depending on the boundary conditions imposed on the element. The error indicator being considered should be normalized using the maximum value for the error indicator for the same quantity on the subregion to which the element belongs. Once the value for the error indicator has been normalized, it can be used as representative for the element.

4. The reference values are relative and the element is lying on an interface. In this case four values for the error indicator are available: $\lambda_{T_{1}}, \lambda_{T_{2}} \lambda_{q_{1}}$, and $\lambda_{q_{2}}$, where the different subindexes are used to indicate that the error indicators belong to different subregions. Although continuity and equilibrium conditions at the interface results in $\lambda_{T_{1}}=\lambda_{T_{2}}$ and $\lambda_{q_{1}}=\lambda_{q_{2}}$, each value should be treated separately since they have to be normalized independently. Each value is normalized using the maximum value of the error indicator for the same quantity on the region to which the error belongs. Once normalized, the four values are compared and the largest is taken as representative for the element.

\subsubsection{Thermoelastic problem}

The handling of element improvement in the thermoelastic problem is simpler since the error indicator is always given in terms of stresses. Special care must be taken at the interfaces since the values for the stresses may be different from one subregion to the other if the subregions have different material properties. Again, in general four cases are to be addressed:

1. The reference value is absolute and the element is lying on the boundary. This is the most straightforward case as only one value for the error indicator in the element is available.

2. The reference value is absolute and the element is lying on an interface. The element has two values for the error indicator, one for each subregion. The one with the larger value is taken as representative for the element.

3. The reference value is relative and the element is lying on the boundary. In this case, the element must be normalized using the maximum value for the error indicator in the subregion to which the element belongs.

4. The reference value is relative and the element is lying on an interface. The element has again two values for the error indicator, one for each subregion. Each value for the error indicator must be normalized using the maximum value of the error indicator corresponding to the elements in the subregion for which the error indicator is considered. The largest normalized value is taken as representative for the element.

If all the subregions in the model have the same material properties, the cases mentioned above can be simplified as the values for the error indicator at the interfaces no longer depend on the subregion to which they belong.

\subsection{Element improvement}

The procedure described above is quite general and can be applied to any element improvement scheme independently if it is $p-, h-, r-$, or any combination of them. In this work, the $h$-method is used and all those elements candidates to be improved are refined in two elements of equal size.

In order to minimize the errors related to the numerical integration, two adjacent elements cannot be much different in size. In this regard, Guiggiani [32] proposed that the ratio of the lengths of two adjacent elements must be kept in the range from 0.25 to 4 . If this compatibility condition for integration is not satisfied, the larger element is refined.

Taking into account that the thermoelastic analysis is carried out once the heat conduction analysis has been performed, it is possible to have different alternatives to specify the way in which the mesh refinement process is 
accomplished. Kamiya et al. [16] present two options:

Method 1 Run the adaptive process for the heat conduction problem for a certain number of times. Then, run the adaptive process for the thermoelastic problem until the selected stop criterion is met.

Method 2 Run alternatively the adaptive process for the heat conduction and the thermoelastic problems until the selected stop criterion is met.

Although both methods give satisfactory results, the second one seems more natural since there is no need to specify a number of runs for the heat conduction problem. Kamiya et al. implemented the second method improving the mesh each time an analysis was run, either heat conduction or thermoelastic. In this scheme, once the error analysis for the heat conduction problem has been performed, a new improved mesh for the thermoelastic problem is modified to match the new heat conduction mesh and the thermoelastic problem is solved. If the stop criteria is not fulfilled, then the mesh should be improved and a new iteration started.

Some enhancement to the process mentioned above can be achieved by improving the mesh only after both error analyses have been performed, that is, just one time per iteration. This can be done solving the heat conduction model and running the error analysis, but not improving the mesh immediately. Instead, a list of the elements that need to be improved is saved. This list is then used together with a similar list for the thermoelastic problem obtained after running the error analysis for this problem. The elements to be improved will be the ones that appear as candidates in any of the two lists. This improved scheme is computationally cheaper since one solution for the heat conduction problem is avoided. Yet, it provides similar results than the scheme presented by Kamiya et al. Fig. 4 shows in a schematic fashion the differences between both methods. As can be observed, the proposed method saves some steps on each iteration, making it also simpler to implement.

\subsection{Stop criteria}

The stop criteria for the adaptive process can be specified based on the thermoelastic model only. This is because usually the analyst is only interested in the values of the stresses. Also, in many cases, the heat conduction problem may converge faster to the analytical solution than the thermoelastic one.

To stop the adaptive process, one of the following options can be used:

1. Specify a maximum value for the average index $\tau$ in the mesh. If for any mesh the average index is smaller than the value specified by the user, the adaptive process is ended. Although this criterion is useful when the user is interested in global accuracy, it is necessary to take into account that a small average index can mask one or more elements with a large error.

2. Specify a maximum absolute value of the error indicator for the elements in the mesh. If for any mesh the error indicator in each one of the elements is smaller than the one specified by the user, the adaptive process is ended.

3. Specify a maximum value for the difference in certain variable of the problem (like displacements or stress) between to consecutive analyses. If the difference is less that the one specified by the user, the process is ended.

In addition, a limit in the number of iterations can be specified for those cases where the adaptive process is unable to fulfill the specified stop criteria after a certain number of iterations.

\section{Numerical examples}

To test the performance of the error indicator, three different problems were selected. The first problem is a thick wall cylinder subject to internal pressure and a temperature distribution. The second one is a square plate with a circular hole subject to a temperature field described by a 4th degree polynomial and a displacement field described by a 5 th degree polynomial. Finally, a case involving a glass mold is analyzed.

\subsection{Example 1: thick wall cylinder}

Consider a thick-walled hollow cylinder with an inner radius $R_{\text {int }}$ and an outer radius $R_{\text {out }}$. The inner surface is maintained at a temperature $T_{\text {int }}$ and is subject to an internal pressure $p_{\text {int }}$. The outer surface is considered free of tractions and is maintained at zero temperature. The ends of the cylinder are assumed to be thermally insulated, which makes the heat conduction purely radial. In addition to this, the cylinder is considered to be long enough for plane strain conditions to apply. Fig. 5 shows the boundary value problem for the example under consideration together with the modeled region. The analytical solution for this problem can be found in Ref. [33].

For this example, and the following ones, the error indicator for the thermoelastic problem, $\lambda_{\sigma}^{(e)}$, was based on the von Mises stress. For the heat conduction problem, the error indicator $\lambda_{m}^{(e)}$ was based on the tangential derivative of the normal heat flux or temperature, depending on which quantity was specified as boundary condition for the element. Error indicators were computed in the $L_{2}$ norm using eight quadrature points.

As improvement criterion, all those elements with a value for the error indicator larger than $5 \%$ the maximum value for the quantity in which they are based $(\mathrm{d} u / \mathrm{d} s, \mathrm{~d} q / \mathrm{d} s$, or $\left.\sigma_{v m}\right)$ were refined in two elements of equal size. The 
Kamiya et al. Method 2

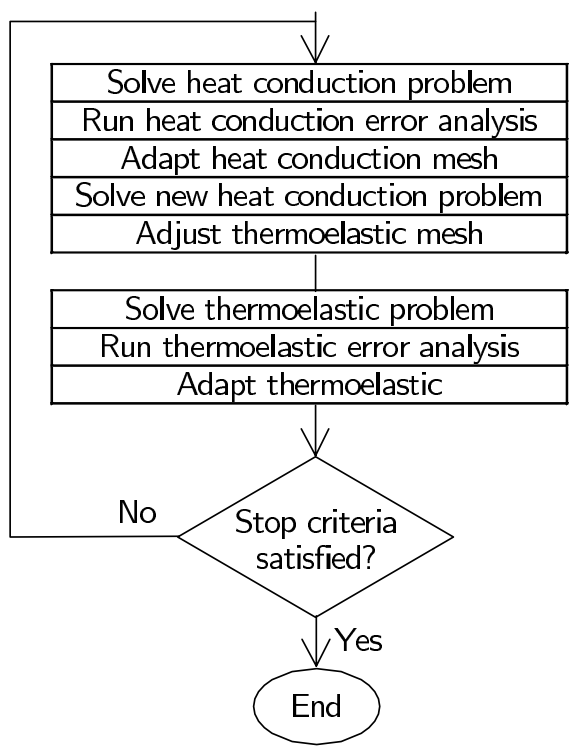

Method proposed here

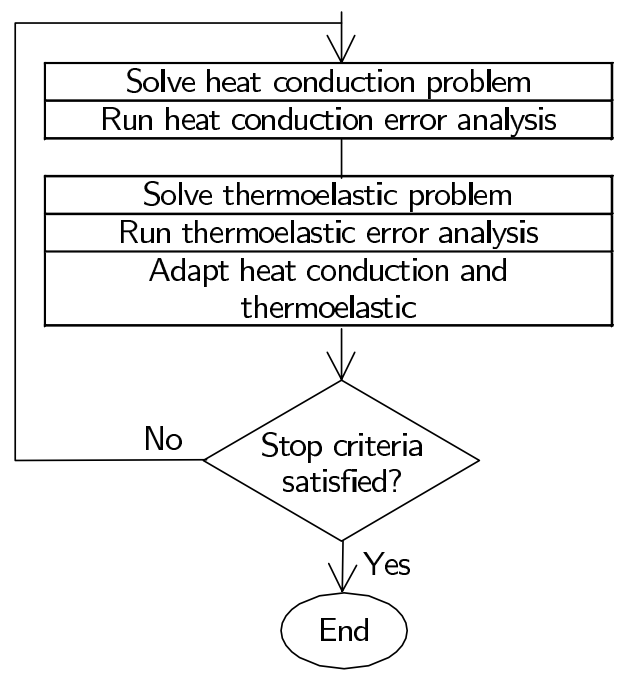

Fig. 4. Comparison between two different adaptive meshing implementations.

adaptive process was stopped when there were no more elements to refine.

Due to the axisymmetric nature of the problem, it should be expected to find most of the refinement in segments $A B$ and CD. Also, the distribution of the elements along these two segments should be symmetric and the mesh distribution should show more elements towards points A and D, which are the points where the maximum von Mises stress occurs. Fig. 6 shows the initial and final meshes for the adaptive process, which took three steps to complete. The mesh refinement distribution shown agrees with the points mentioned above. Here it should be mentioned that the refinement that took place on the boundary $R=R_{\text {int }}$ was only due to the enforcement of the compatibility condition for integration.

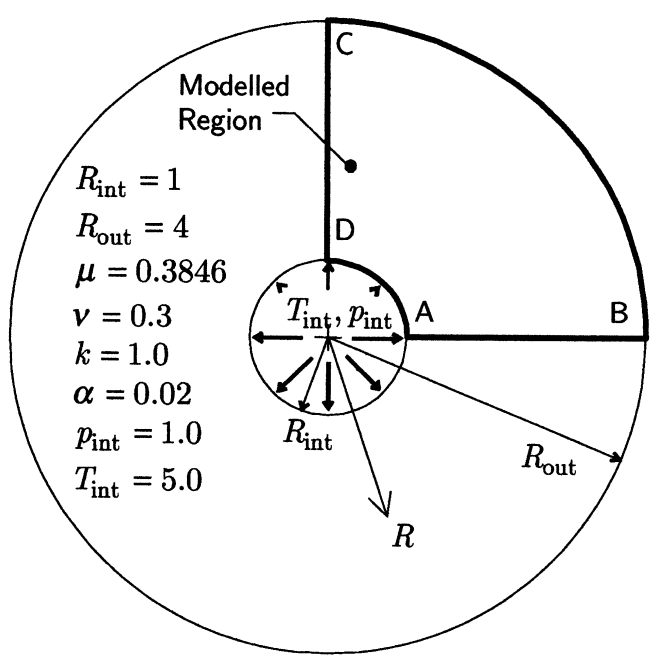

Fig. 5. Boundary value problem for Example 1.
Fig. 7 compares the behavior of the error indicator against the true error along the segment $\mathrm{AB}$ for the initial and final meshes. From the plots it is evident that the error indicator is over-predicting the actual error in the model. Although this is not desirable, the error indicator seems to point in an effective way those areas with the larger error, refining the mesh accordingly.

Fig. 8 shows the behavior of the maximum value of $\lambda_{\sigma}^{(e)}$ and the maximum absolute value of the true error in the von Mises stress during the adaptive process. As can be observed, both the maximum error indicator and the maximum true error tend to zero as the mesh is refined.

\subsection{Example 2: square plate with a circular hole}

The second example is a square plate with a circular hole. The plate is subject to a temperature field that is described by a 4th degree polynomial and a displacement field corresponding to a 5th degree polynomial. The plate has the following properties: $E=70$, $\nu=0.33, \alpha=23 \times 10^{-6}$, and $k=3.86$. The value of the temperature was prescribed as boundary condition all over the boundary. Displacements and tractions were prescribed in different parts of the boundary as shown in Fig. 9.

Boundary conditions are applied in such a way that the analytical solution is given by the following polynomials:

$$
\begin{aligned}
T\left(x_{1}, x_{2}\right)= & c_{1}+c_{2} x_{1}+c_{3} x_{2}+c_{4} x_{1} x_{2}+c_{5}\left(x_{1}^{2}-x_{2}^{2}\right) \\
& +c_{6}\left(x_{1}^{3}-3 x_{1} x_{2}^{2}\right)+c_{7}\left(x_{2}^{3}-3 x_{1}^{2} x_{2}\right) \\
& +c_{8}\left(x_{1}^{4}-6 x_{1}^{2} x_{2}^{2}+x_{2}^{4}\right)+c_{9}\left(x_{1}^{3} x_{2}-x_{1} x_{2}^{3}\right)
\end{aligned}
$$



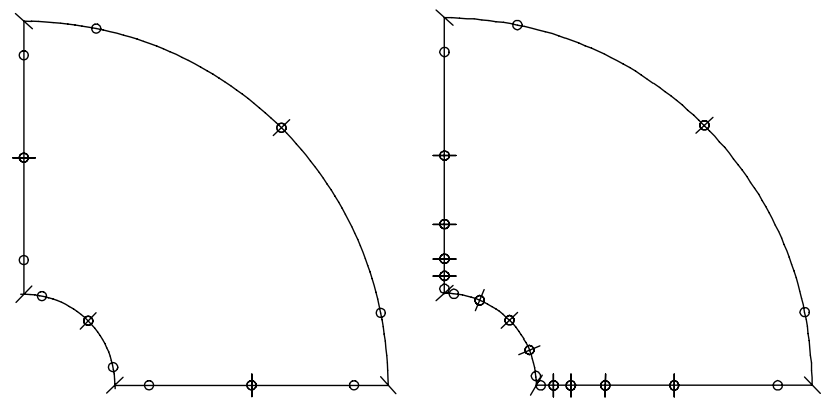

Fig. 6. Initial and final meshes for Example 1. The meshes have 8 and 16 elements, respectively.

$$
\begin{aligned}
u_{1}\left(x_{1}, x_{2}\right)= & a_{1}+a_{2} x_{1}+a_{4} x_{1}^{2}+a_{7} x_{1}^{3}+a_{3} x_{2}+a_{5} x_{1} x_{2} \\
& +a_{8} x_{1}^{2} x_{2}+a_{12} x_{1}^{3} x_{2}+a_{6} x_{2}^{2}+a_{9} x_{1} x_{2}^{2}+a_{10} x_{2}^{3} \\
& +a_{13} x_{1} x_{2}^{3}+a_{11}\left(x_{1}^{4}-3 x_{1}^{2} x_{2}^{2}\right)+a_{15}\left(x_{1}^{5}-5 x_{1}^{3} x_{2}^{2}\right) \\
& +a_{16}\left(x_{1}^{4} x_{2}-x_{1}^{2} x_{2}^{3}\right)+a_{14}\left(-3 x_{1}^{2} x_{2}^{2}+x_{2}^{4}\right) \\
& +a_{17}\left(-x_{1}^{3} x_{2}^{2}+x_{1} x_{2}^{4}\right)+a_{18}\left(-5 x_{1}^{2} x_{2}^{3}+x_{2}^{5}\right)
\end{aligned}
$$

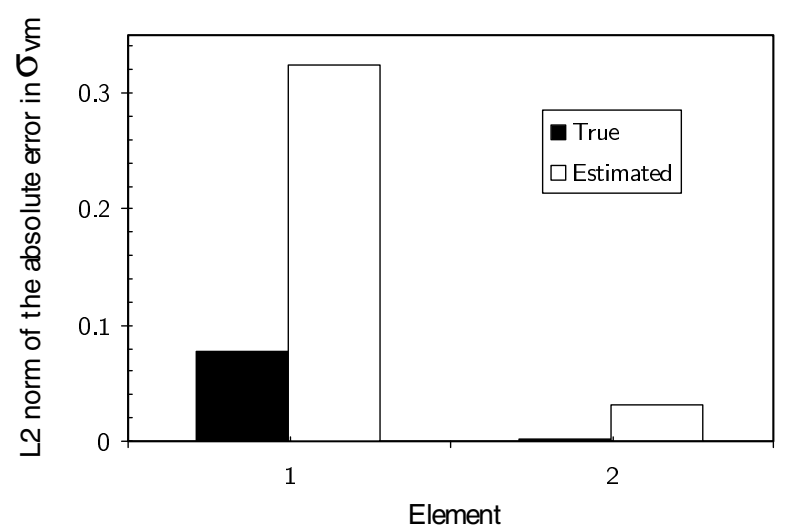

(a)

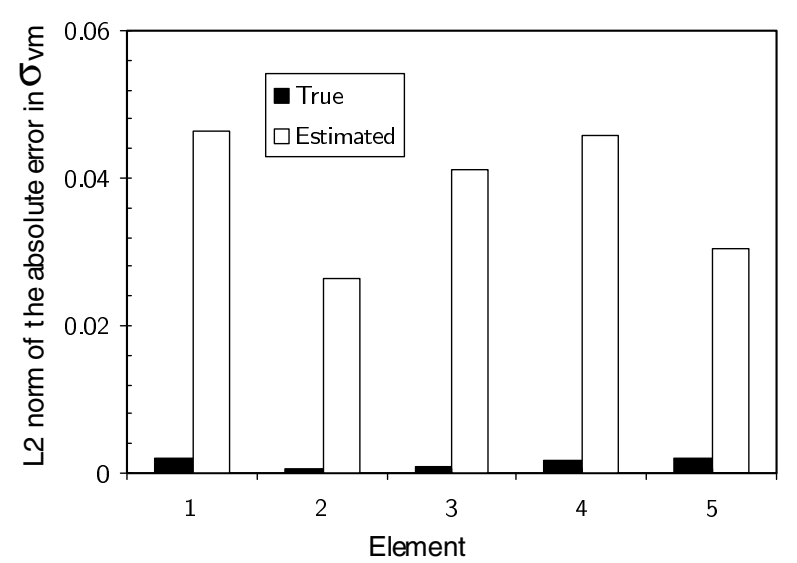

(b)

Fig. 7. Comparison of the absolute errors in $\sigma_{v m}$ along segment $\mathrm{AB}$ for the (a) initial and (b) final meshes.

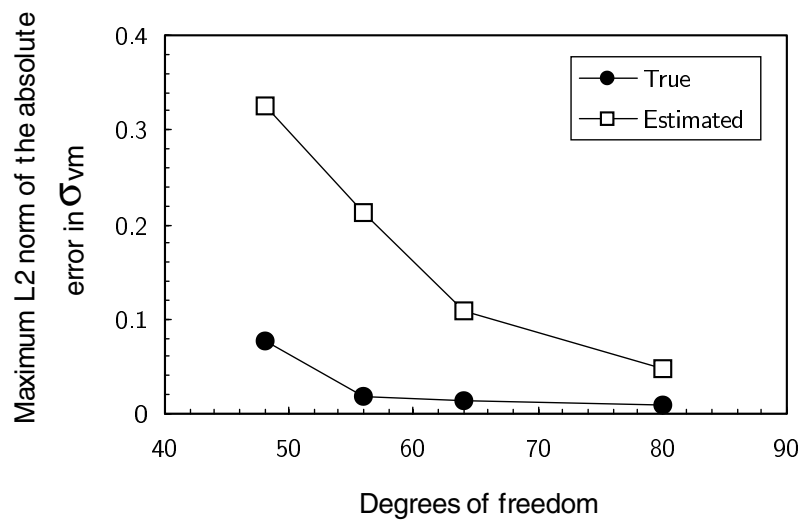

Fig. 8. Behavior of the error indicator $\lambda_{\sigma}^{(e)}$ and the maximum true error during the adaptive process.

$$
\begin{aligned}
u_{2}\left(x_{1}, x_{2}\right)= & a_{19}+a_{20} x_{1}-\frac{a_{5}}{2 d_{1}} x_{1}^{2}+\frac{c_{3} d_{2}}{2 d_{1}} x_{1}^{2}+a_{21} x_{2} \\
& -2 a_{6} d_{1} x_{1} x_{2}-2 a_{4}\left(1+d_{1}\right) x_{1} x_{2}+c_{2} d_{2} x_{1} x_{2} \\
& +a_{22}\left[x_{2}^{2}-\frac{1+d_{1}}{d_{1}} x_{1}^{2}\right]+a_{10}\left[\left(1+d_{1}\right) x_{1}^{3}\right. \\
& \left.-3 d_{1} x_{1} x_{2}^{2}\right]+a_{8}\left[\frac{2+d_{1}}{3} x_{1}^{3}-\left(1+d_{1}\right) x_{1} x_{2}^{2}\right] \\
& +c_{4}\left[-\frac{d_{2}}{6} x_{1}^{3}+\frac{d_{2}}{2} x_{1} x_{2}^{2}\right] \\
& +a_{9}\left[\frac{d_{1}-1}{3} x_{2}^{3}-d_{1} x_{1}^{2} x_{2}\right] \\
& +a_{7}\left[-3\left(1+d_{1}\right) x_{1}^{2} x_{2}+d_{1} x_{2}^{3}\right] \\
& +c_{5}\left[d_{2} x_{1}^{2} x_{2}-\frac{d_{2}}{3} x_{2}^{3}\right] \\
& +a_{14}\left[2 d_{1} x_{1}^{3} x_{2}+2\left(1-d_{1}\right) x_{1} x_{2}^{3}\right] \\
& +a_{11}\left[-2\left(2+d_{1}\right) x_{1}^{3} x_{2}+2\left(1+d_{1}\right) x_{1} x_{2}^{3}\right] \\
& +c_{6}\left[d_{2} x_{1}^{3} x_{2}-d_{2} x_{1} x_{2}^{3}\right] \\
& +a_{13}\left[\frac{1+d_{1}}{4} x_{1}^{4}-\frac{3 d_{1}}{2} x_{1}^{2} x_{2}^{2}-\frac{1-d_{1}}{4} x_{2}^{4}\right]
\end{aligned}
$$

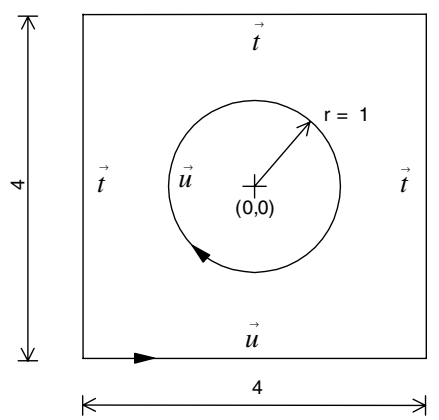

Fig. 9. Boundary value problem for Example 2. 


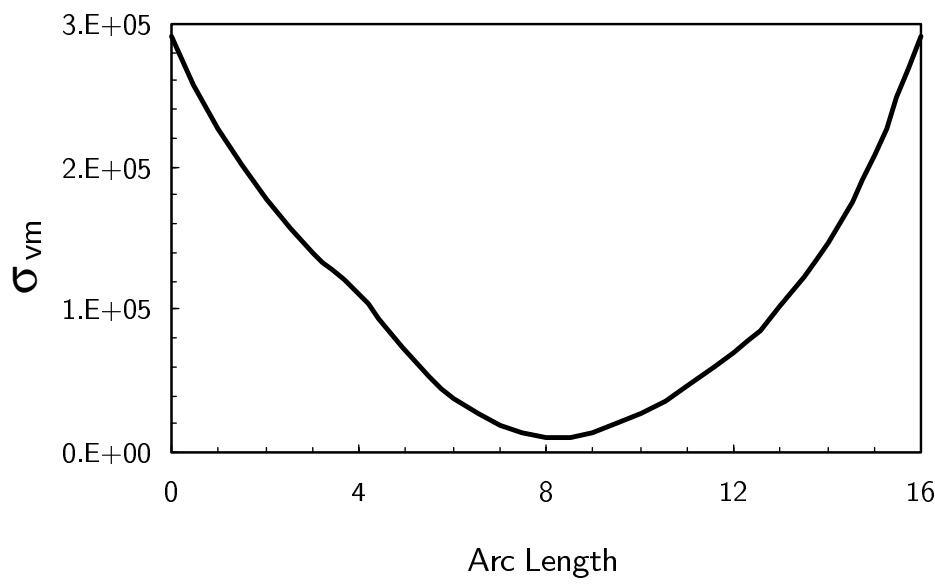

(a)

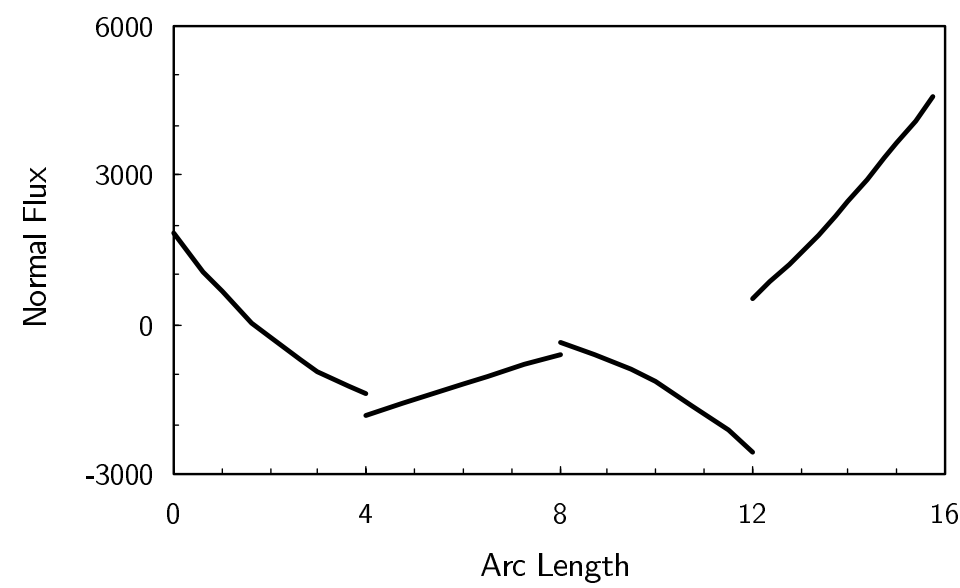

(c)

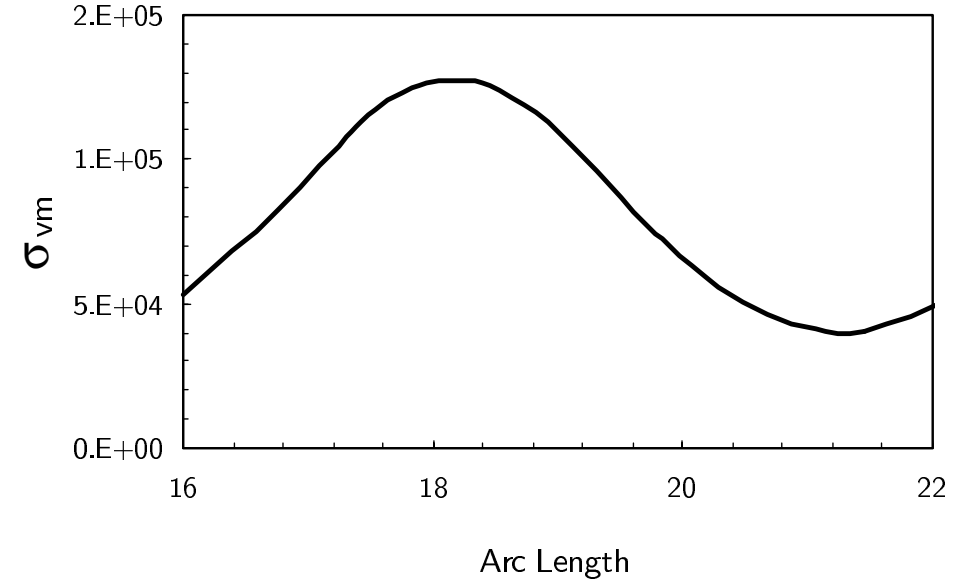

(b)

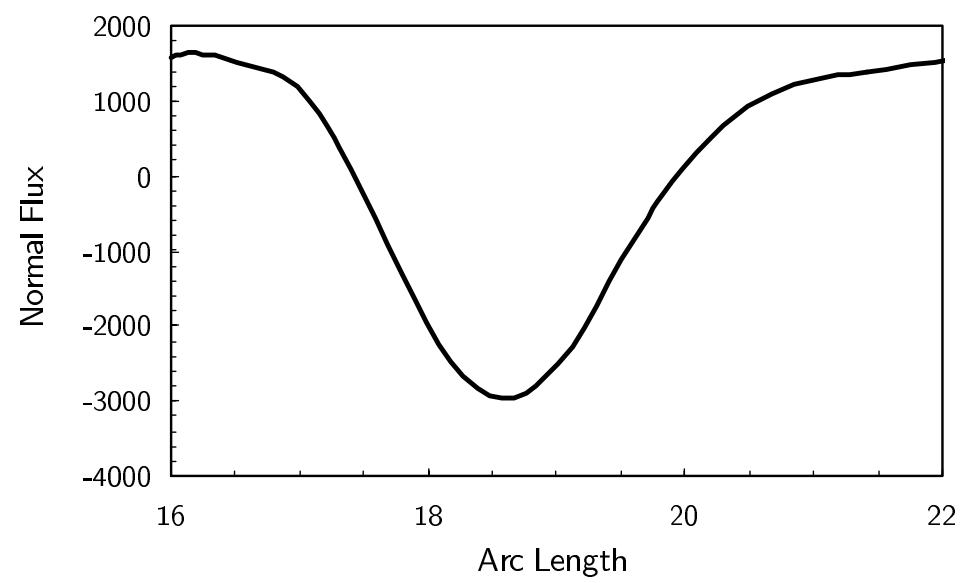

(d)

Fig. 10. Analytical solution for the flux and von Mises stress for the problem of Example 2. (a) and (c), external boundary. (b) and (d), internal boundary. 

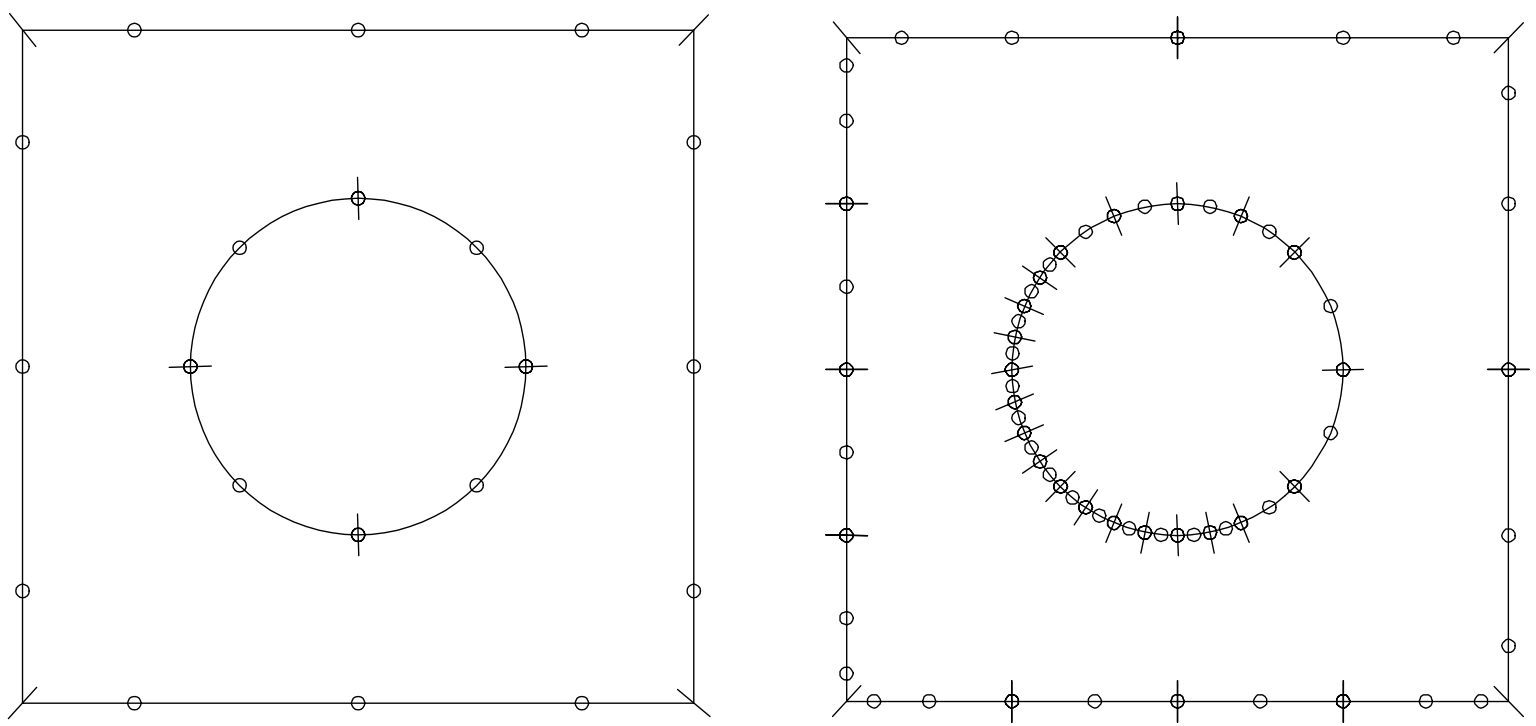

Fig. 11. Initial and final meshes of the adaptive process for Example 2. The meshes have 8 and 33 elements, respectively.

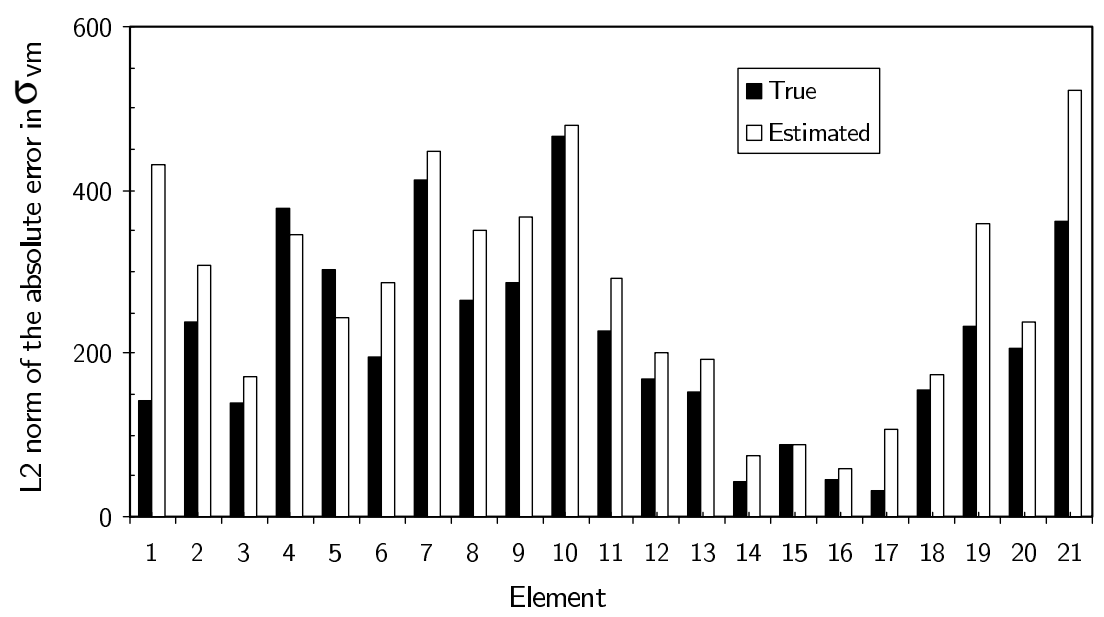

Fig. 12. Comparison of the absolute errors in $\sigma_{v m}$ along the circular boundary for the last mesh.

$$
\begin{aligned}
& +a_{12}\left[\frac{2+d_{1}}{4} x_{1}^{4}-\frac{3\left(1+d_{1}\right)}{2} x_{1}^{2} x_{2}^{2}+\frac{d_{1}}{4} x_{2}^{4}\right] \\
& +c_{7}\left[\frac{d_{2}}{4} x_{1}^{4}-\frac{3 d_{2}}{2} x_{1}^{2} x_{2}^{2}+\frac{d_{2}}{4} x_{2}^{4}\right] \\
& +a_{18}\left[\frac{-\left(1+d_{1}\right)}{2} x_{1}^{5}+5 d_{1} x_{1}^{3} x_{2}^{2}+\frac{5\left(1-d_{1}\right)}{2} x_{1} x_{2}^{4}\right] \\
& +a_{16}\left[\frac{3+d_{1}}{10} x_{1}^{5}-\left(2+d_{1}\right) x_{1}^{3} x_{2}^{2}+\frac{1+d_{1}}{2} x_{1} x_{2}^{4}\right] \\
& +c_{9}\left[-\frac{d_{2}}{20} x_{1}^{5}+\frac{d_{2}}{2} x_{1}^{3} x_{2}^{2}-\frac{d_{2}}{4} x_{1} x_{2}^{4}\right] \\
& +a_{17}\left[\frac{d_{1}}{2} x_{1}^{4} x_{2}+\left(1-d_{1}\right) x_{1}^{2} x_{2}^{3}+\frac{-2+d_{1}}{10} x_{2}^{5}\right] \\
& +a_{15}\left[\frac{-5\left(2+d_{1}\right)}{2} x_{1}^{4} x_{2}+5\left(1+d_{1}\right) x_{1}^{2} x_{2}^{3}-\frac{d_{1}}{2} x_{2}^{5}\right]
\end{aligned}
$$$$
+c_{8}\left[d_{2} x_{1}^{4} x_{2}-2 d_{2} x_{1}^{2} x_{2}^{3}+\frac{d_{2}}{2} x_{2}^{5}\right]
$$

where $d_{1}=1-2 \nu, d_{2}=2 \alpha(1+\nu)$ and constants $c_{1}$ to $c_{9}$ and $a_{1}$ to $a_{22}$ are given in Appendix A.

For this problem, the improvement criteria was to refine into two elements of equal size all those elements with a value for the error indicator larger than the average value for the same quantity. To show some of the flexibility of the error indicator formulation, this example was modeled using Hermite elements with three nodes. The adaptive process was stopped after three steps.

In this problem, the gradients of the analytical solution for the normal heat flux and the von Mises stress along the circular boundary present a more involved behavior than along the exterior boundary of the model (see Fig. 10). Therefore, it is expected that the refinement should take place mainly in the circular boundary. Fig. 11 shows the 


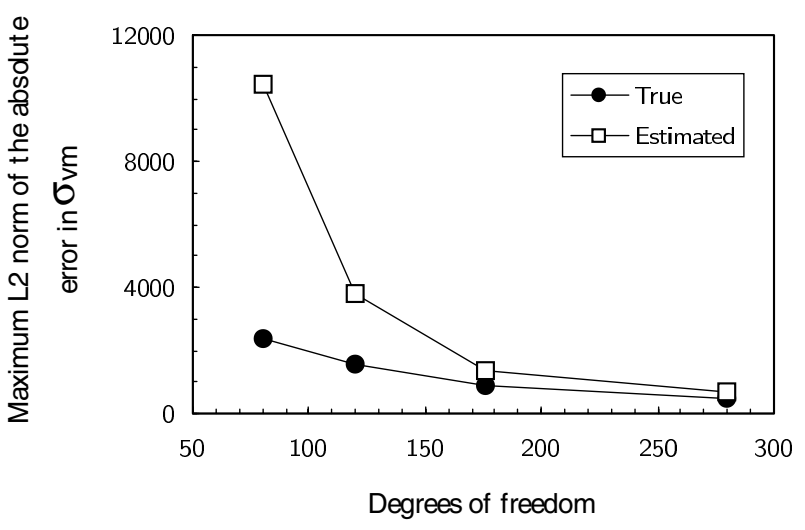

Fig. 13. Convergence to the analytical solution in the adaptive process for Example 2.

initial and final meshes for the adaptive process. It is clear from this figure that the most of the refinement took place on the circular boundary.

Fig. 12 compares the behavior of the true and estimated error in the von Mises stress for the elements lying along the circular boundary of the final mesh. It can be easily appreciated that, although the error indicator fails to estimate the error correctly in some elements, in general the error indicator provides a fairly good approximation. Taking into account the low computational cost of the error indicator, its performance is quite attractive for driving adaptive processes.

In Fig. 13 the behavior of the maximum value of $\lambda_{\sigma}^{(e)}$ and the maximum value of the true error in the von Mises stress during the adaptive process is shown. As should be expected, both quantities diminish with each iteration and become closer to each other as the mesh is refined.

\subsection{Example 3: glass mold}

The third example is a simplified model of a glass mold. In the glass molding process it is necessary to keep the mold cool to avoid permanent deformation. This is generally achieved by drilling cooling holes in the mold. In this example, both the glass and the mold will be modeled considering the following simplifications:

- A steady state, plane strain problem.

- The glass has a linear behavior.

- The glass and the mold are perfectly coupled.

- The cooling holes are kept at a constant temperature.

The problem is modeled using two subregions, one for the cast iron mold and one for the glass (see Fig. 14). The material properties considered are as follows. For the glass: $E=80 \mathrm{GPa}, \nu=0.27, \alpha=6 \times 10^{-6} \mathrm{~K}^{-1}$, and $k=$ $1.4 \mathrm{~W} / \mathrm{mK}$. For the mold: $E=175 \mathrm{GPa}, \nu=0.26, \alpha=$ $13 \times 10^{-6} \mathrm{~K}^{-1}$, and $k=10 \mathrm{~W} / \mathrm{mK}$. For the discretization of the boundary, Hermite elements with two nodes were used to approximate the field variables, whereas linear and cubic Lagrangian elements were used to approximate the geometry.

The initial and final meshes for this problem are shown in Fig. 15. For this problem the improvement criteria was to refine in two elements of equal size all those element with a

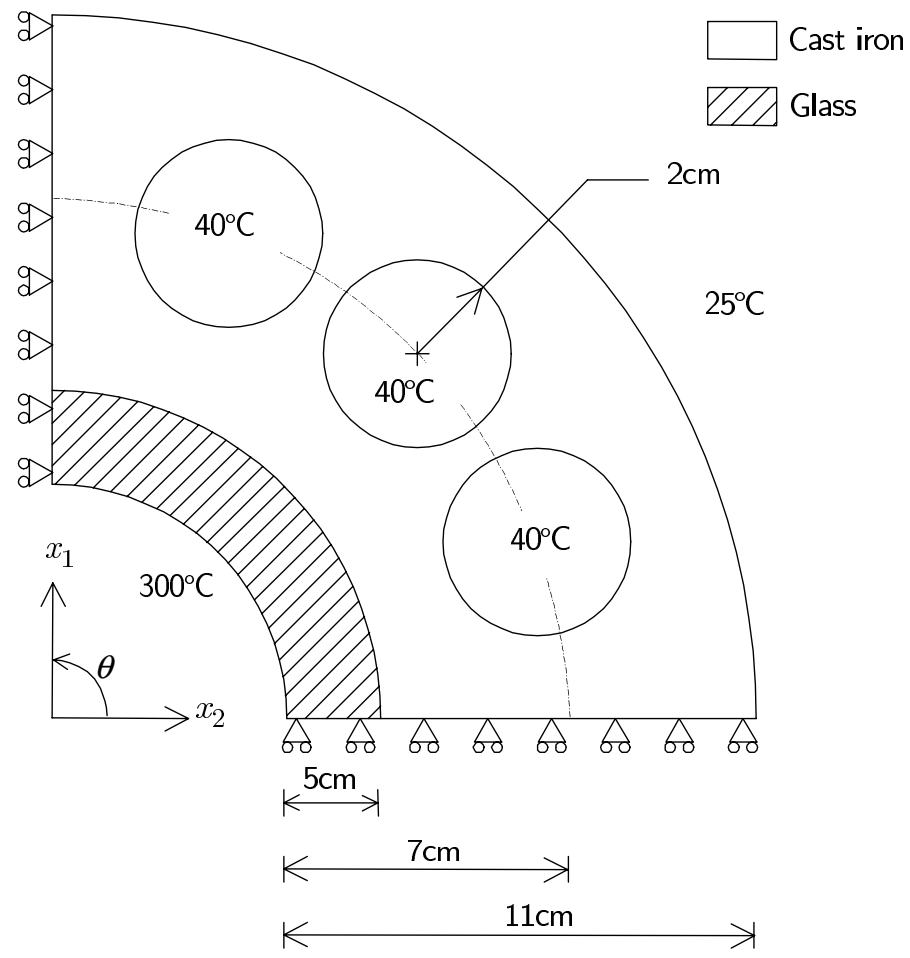

Fig. 14. Boundary value problem for Example 3. 

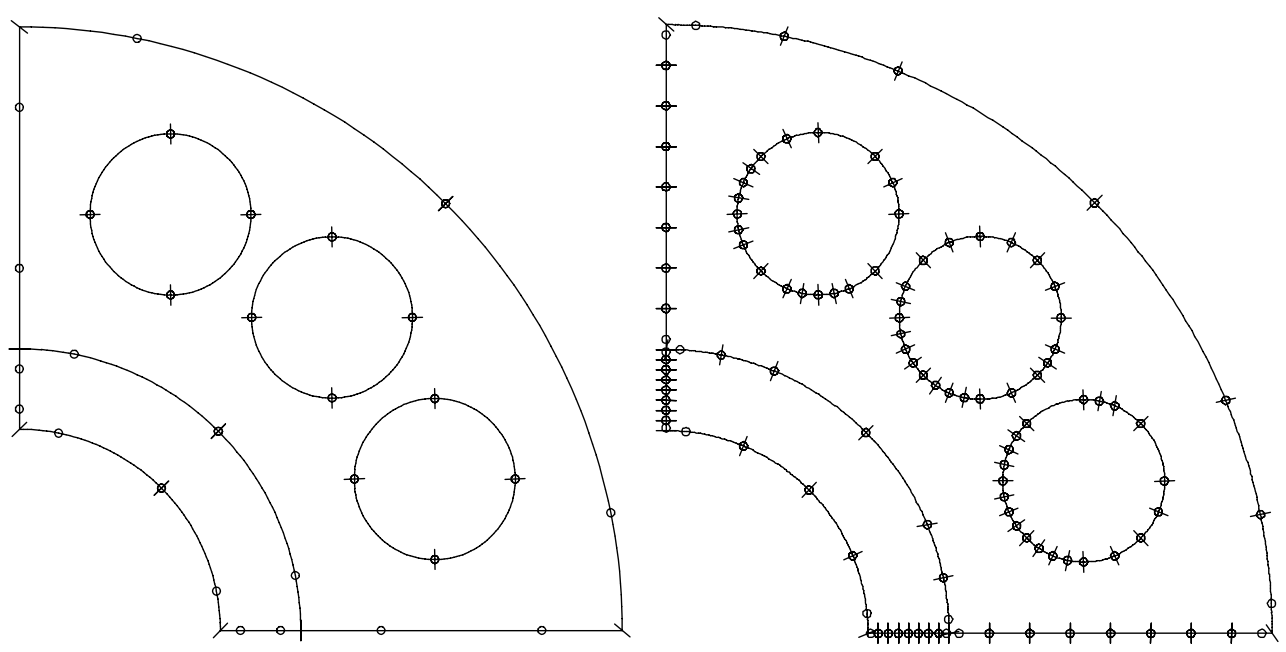

Fig. 15. Initial and final meshes of the adaptive process for Example 3. The meshes have 22 and 110 elements, respectively.

value for the error indicator larger than the average value for the same quantity. For this particular problem the "compatibility condition for integration" was not applied in order to consider the refinement due to the error distribution only. The adaptive process was stopped after three steps.

From the final mesh it can be observed that the refinement took place along the boundaries of the cooling holes and on the boundaries $x_{1}=0$ and $x_{2}=0$. There is also some refinement at the interface between the glass and the mold. The refinement along the boundaries of the cooling holes is due to the high gradients in the normal heat flux that these boundaries have. Although the refinement of the three holes should be symmetric with respect to the line $\theta=$ $45^{\circ}$, small errors in the approximation of the geometry seem to affect the computation of the error indicator, creating a slightly different refinement pattern. The refinement along the boundaries $x_{1}=0$ and $x_{2}=0$ is symmetric as should be expected with finer elements in the glass region where the gradients in the temperature and von Mises stress are higher. Fig. 16 shows the behavior of the maximum value for the error indicator at each step of the adaptive process.

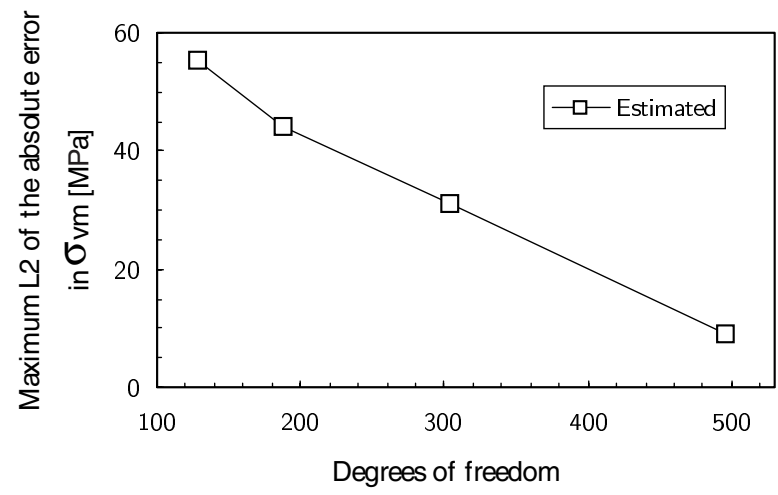

Fig. 16. Behavior of the maximum value for the error indicator at each step of the adaptive process for Example 3.

\section{Conclusions}

In this work, an alternative for the implementation of adaptive meshing for two-dimensional thermoelastic problems using Hermite elements was presented. The fact that Hermite elements provide a convenient formulation for the computation of error indicators was exploited to lead adaptive processes aimed at improving the quality of the numerical solution obtained by the BEM. Two different error indicators were used since the thermoelastic analysis in its uncoupled fashion was considered. This approach gave the opportunity to measure the error in the heat conduction and thermoelastic solutions separately, which keeps the process simple. For the heat conduction problem, a new more general formulation for the computation of error indicators for Hermite elements was developed. In addition, a convenient alternative in the way in which the adaptive process may be controlled has been presented. This scheme is simpler and computationally cheaper than other alternatives and yet provides similar results to the ones that are obtained using schemes that are more sophisticated. Finally, all the error analysis is done as a post-processing activity which makes it possible to implement the formulation presented here in existing codes that use Hermite elements without the need of extensive modifications to them.

\section{Acknowledgements}

J.C. Miranda-Valenzuela acknowledges the financial support provided by CONACyT, the Mexican council for science and technology, for the realization of this work.

\section{Appendix A}

The temperature distribution in Eq. (45) satisfies the Laplace equations if the following values for the constants 
$c_{1}$ to $c_{9}$ are considered:

$$
\begin{array}{lll}
c_{1}=1202.91 & c_{2}=509.612 & c_{3}=218.803 \\
c_{4}=178.535 & c_{5}=437.947 & c_{6}=2.75 \\
c_{7}=8.50107 & c_{8}=0.46933 & c_{9}=0.27756
\end{array}
$$

The displacement distribution in Eqs. (46) and (47) satisfy Navier's equations if the temperature distribution in Eq. (45) is employed, the following material properties are used

$\nu=0.33 \quad \alpha=23 \times 10^{-6}$

and the following values for the constants $a_{1}$ to $a_{22}$ are considered:

$$
\begin{array}{lcc}
a_{1}=1794.06 & a_{2}=-936.629 & a_{3}=-326.44 \\
a_{4}=143.449 & a_{5}=417.452 & a_{6}=-117.036 \\
a_{7}=-4.53626 & a_{8}=-76.5294 & a_{9}=-43.7022 \\
a_{10}=32.8545 & a_{11}=-0.506191 & a_{12}=3.70973 \\
a_{13}=0.0654636 & a_{14}=-2.72739 & a_{15}=0.0340007 \\
a_{16}=-0.064768 & a_{17}=0.0870474 \\
a_{18}=0.0779335 & a_{19}=-887.189 \\
a_{21}=684.417 & a_{22}=-150.395
\end{array}
$$

\section{References}

[1] Watson JO. Hermitian cubic and singular elements for plane strain. In: Banerjee PK, Watson JO, editors. Developments in boundary element methods, vol. 4. London: Elsevier Applied Science, 1986.

[2] Rudolphi TJ. Higher order elements and Element Enhancement by combined regular and hypersingular boundary integral equations. Paper presented at the International Symposium on Boundary Element Methods, Connecticut, USA, 1989.

[3] Durodola JF, Fenner RT. Hermitian cubic boundary elements for twodimensional potential problems. International Journal for Numerical Methods in Engineering 1990;30:1051-62.

[4] Rudolphi TJ. The use of simple solutions in the regularization of hypersingular integral equations. Mathematical Computer Modelling 1991;15:269-78.

[5] Muci-Küchler KH, Rudolphi TJ. Coincident collocation of displacement and tangent derivative boundary integral equations in elasticity. International Journal for Numerical Methods in Engineering 1993;36:2837-49.

[6] Gray LJ, San Soucie CA. A Hermite interpolation algorithm for hypersingular boundary integrals. International Journal for Numerical Methods in Engineering 1993;36:2357-67.

[7] Muci-Küchler KH, Rudolphi TJ. Application of tangent derivative boundary integral equations to the formulation of higher order boundary elements. International Journal of Solids and Structures 1994;31:1565-84

[8] Pullan AJ, Bradley CP. A coupled cubic hermite finite element/ boundary element procedure for electrocardiographic problems. Computational Mechanics 1996;18:356-68.

[9] Becker AA. The boundary element method in engineering - a complete course. New York: McGraw-Hill, 1992.

[10] Banerjee AA. The boundary element method in engineering. Berkshire: McGraw-Hill, 1994.

[11] Muci-Küchler KH, Hernández-Luna JA. Formulation of higher order boundary elements for the solution of steady state thermoelastic problems. Paper presented at the Twenty-fourth Midwestern Mechanics Conference. Ames, IA, USA, 1995.

[12] Prasad NNV. Thermomechanical crack growth using boundary elements. Southampton: WIT Press, 1999.

[13] dell'Erba DN, Aliabadi MH, Rooke DP. Dual boundary element method for three-dimensional thermoelastic crack problems. International Journal of Fracture 1999;94:89-101.

[14] Kita E, Kamiya N. Recent studies on adaptive boundary element methods. Advances in Engineering Software 1994;19:21-32.

[15] Liapis S. A review of error estimation and adaptivity in the boundary element method. Engineering Analysis with Boundary Elements 1994;14:315-23.

[16] Kamiya N, Aikawa Y, Kawaguchi K. An adaptive boundary element scheme for steady thermoelastostatic analysis. Computational Methods in Applied Mechanics and Engineering 1994;119:311-24.

[17] Kamiya N, Koide N. Adaptive boundary element for multiple subregions. Computational Mechanics 1993;12:69-80.

[18] Kamiya N, Koide N, Kawaguchi K. Adaptive boundary elements for elastic analysis of multiple subregions. Advances in Engineering Software 1993;17:125-34.

[19] Muci-Küchler KH, Miranda-Valenzuela JC. A new error indicator based on stresses. Engineering Analysis with Boundary Elements 1999;23:657-70.

[20] Charafi A, Neves AC, Wrobel LC. h-Hierarchical adaptive boundary element method using local reanalysis. International Journal for Numerical Methods in Engineering 1995;38:2185-207.

[21] Guiggiani M. Sensitivity analysis for boundary element error estimation and mesh refinement. International Journal for Numerical Methods in Engineering 1996;39:2907-20.

[22] Paulino GH, Shi F, Mukherjee S, Ramesh P. Nodal sensitivities as error estimates in computational mechanics. Acta Mechanica 1997;121:191-213.

[23] Gallego R, Martínez-Castro A, Suarez J. Sensitivity analysis of approximate solutions for the hypersingular boundary element method. Paper presented at the Boundary Elements Techniques Conference. London, UK, 1999.

[24] Paulino GH, Gray LJ, Zarikian V. Hypersingular residuals - a new approach for error estimation in the boundary element method. International Journal for Numerical Methods in Engineering 1996;39:2005-29.

[25] Liang MT, Chen JT, Yang SS. Error estimation for boundary element method. Engineering Analysis with Boundary Elements 1999;23:257-65.

[26] Menon G, Paulino GH, Mukherjee S. Analysis of hypersingular residuals error estimates in boundary element methods for potential problems. Computational Methods in Applied Mechanics and Engineering 1999;173:449-73.

[27] Muci-Küchler KH, Miranda-Valenzuela JC, Rudolphi TJ. A new error indicator for adaptive meshing with Hermite boundary elements. Computational Methods in Applied Mechanics and Engineering 1999;173:419-31.

[28] Rencis JJ, Mullen RL. Solution of elasticity problems by a self-adaptive mesh refinement technique for boundary element computation. International Journal for Numerical Methods in Engineering 1986;23:1509-27.

[29] Rencis JJ, Mullen RL. A self adaptive mesh refinement technique. Computational Mechanics 1988;3:309-19.

[30] Rodríguez JJ, Power H. A mesh refinement technique for the boundary element method based on local error analysis. Paper 
presented at the Boundary Elements XXI Conference. Cambridge, UK, 1999.

[31] Miranda-Valenzuela JC, Muci-Küchler KH, Soriano-Soriano S. Adaptive meshing in thermoelasticity using Hermite boundary elements. Paper presented at the Boundary Elements Techniques Conference. London, UK, 1999.
[32] Guiggiani M. Error indicators for adaptive mesh refinement in the boundary element method - a new approach. International Journal for Numerical Methods in Engineering 1990;29:1247-69.

[33] Timoshenko SP, Goodier JN. Theory of elasticity. New York: McGraw-Hill, 1970. 